\title{
Fourier multipliers for Triebel-Lizorkin spaces on compact Lie groups
}

\author{
Duván Cardona ${ }^{1} \cdot$ Michael Ruzhansky ${ }^{1,2}$
}

Received: 14 March 2021 / Accepted: 9 July 2021 / Published online: 6 October 2021

(c) The Author(s) 2021

\begin{abstract}
We investigate the boundedness of Fourier multipliers on a compact Lie group when acting on Triebel-Lizorkin spaces. Criteria are given in terms of the Hörmander-Mihlin-Marcinkiewicz condition. In our analysis, we use the difference structure of the unitary dual of a compact Lie group. Our results cover the sharp Hörmander-Mihlin theorem on Lebesgue spaces and also other historical results on the subject.
\end{abstract}

Keywords Fourier multipliers · Spectral multipliers · Compact Lie groups · HörmanderMihlin theorem $\cdot$ Marcinkiewicz condition $\cdot$ Triebel-Lizorkin spaces

Mathematics Subject Classification $43 \mathrm{~A} 15 \cdot 43 \mathrm{~A} 22 \cdot 22 \mathrm{E} 25 \cdot 43 \mathrm{~A} 80$

\section{Introduction}

Let $G$ be a compact Lie group. In this work we study sufficient conditions for the boundedness of Fourier multipliers on the Triebel Lizorkin spaces $F_{p, q}^{r}(G)$ in terms of the Hörmander-Mihlin condition on their symbols. The Littlewood-Paley theorem states that $L^{p}(G) \equiv F_{p, 2}^{0}(G)$, so that in view of the classical results of Hörmander-Mihlin type (see Hörmander [34] and Mihlin [36] for instance), Triebel-Lizorkin spaces are a good substitute of $L^{p}$-spaces, when considering smoothness of distributions in different scales (see Triebel $[50,51]$ and $[37,38]$ for details).

The authors are supported by the FWO Odysseus 1 grant G.0H94.18N: Analysis and Partial Differential Equations and by the Methusalem programme of the Ghent University Special Research Fund (BOF) (Grant number 01M01021). Michael Ruzhansky is also supported by EPSRC grant EP/ R003025/2.

Duván Cardona

duvanc306@gmail.com; Duvan.CardonaSanchez@ugent.be

Michael Ruzhansky

michael.ruzhansky@ugent.be; m.ruzhansky@qmul.ac.uk

1 Department of Mathematics, Analysis, Logic and Discrete Mathematics, Ghent University, Ghent, Belgium

2 School of Mathematical Sciences, Queen Mary University of London, London, UK 
The problem of finding conditions for the boundedness of Fourier multipliers on compact Lie groups started a long of time ago with the study of the theory of Fourier series of periodic functions on $[0,1]$, (functions on the torus $\mathbb{T}=\mathbb{R} / \mathbb{Z}$ ). Indeed, when classifying the boundedness of multipliers of the Fourier series,

$$
A f(x):=\sum_{\xi \in \mathbb{Z}} e^{2 \pi i x \cdot \xi} \sigma(\xi) \widehat{f}(\xi), \quad f \in L^{1}(\mathbb{T}), \widehat{f}(\xi):=\int_{0}^{1} e^{-2 \pi i x \cdot \xi} f(x) d x,
$$

it was observed by Marcinkiewicz in his classical 1939's work [35] that the condition

$$
\sup _{\xi \in \mathbb{Z}}|\sigma(\xi)|+\sup _{j \in \mathbb{N}_{0}} \sum_{2^{j-1} \leq|\xi|<2^{j}}|\sigma(\xi+1)-\sigma(\xi)|<\infty,
$$

assures the existence of a bounded extension of $A$ on $L^{p}(\mathbb{T}), 1<p<\infty$. Denoting the difference operator on the lattice $\mathbb{Z}$, by $\Delta \sigma:=\sigma(\cdot+1)-\sigma$, and by $\Delta^{k}, k \in \mathbb{N}_{0}$, its successive iterations, the Marcinkiewicz condition (1.2) is satisfied by any sequence $(\sigma(\xi))_{\xi \in \mathbb{Z}^{n}}$ such that

$$
\left|\Delta^{k} \sigma(\xi)\right| \lesssim_{k}|\xi|^{-k}, \quad \xi \neq 0, k=0,1,
$$

which should be, in principle, more easier to verify that (1.2). Another generalisation of Marcinkiewicz's criterion for multipliers of the Fourier transform on $\mathbb{R}^{n}$, was done by Mihlin [36], who stated that a function $\sigma \in C^{\infty}\left(\mathbb{R}^{n} \backslash\{0\}\right)$, satisfying estimates of the kind

$$
\left|\partial_{\xi}^{\alpha} \sigma(\xi)\right| \lesssim_{\alpha}|\xi|^{-|\alpha|}, \quad|\alpha| \leq[n / 2]+1,
$$

has a multiplier $A$ (of the Fourier transform ${ }^{1}$ on $\mathbb{R}^{n}$ ) defined by

$$
A f(x) \equiv T_{\sigma} f(x):=\int_{\mathbb{R}^{n}} e^{2 \pi i x \cdot \xi} \sigma(\xi) \hat{f}(\xi) d \xi, f \in C_{0}^{\infty}\left(\mathbb{R}^{n}\right),
$$

admitting a bounded extension on $L^{p}\left(\mathbb{R}^{n}\right)$, for $1<p<\infty$. Subsequent generalisations to Mihlin's theorem were done by Hörmander [34], Calderón and Torchinsky in [4], Taibleson and Weiss [49], Baernstein and Sawyer [3], Seeger [45-47] and many others. We refer the reader to Grafakos' paper [30] (and reference therein) for a complete historical revision and for recent developments about Milhin-Hörmander and Marcinkiewicz multiplier theorems on $\mathbb{R}^{n}$.

Extensions of Marcinkiewicz, and Hörmander-Mihlin criteria have been proved in the context of Lie groups and several spaces of homogeneous type in the context of spectral multipliers of self-adjoint operators, e.g., sub-Laplacians, or of other operators with heat kernels satisfying Gaussian estimates, with general contexts that go beyond of the objective of this paper. In view of the extensive literature on the field, we will not review it here, but we refer the reader to $[1,2,7,13,14,19,48,53]$ and to the extensive list of references therein.

In the framework of Fourier multipliers on compact Lie groups, by using the Calderón-Zygmund type theory in Coifman and De Guzmán [16], the $L^{p}$-Fourier multipliers for

\footnotetext{
$\overline{{ }^{1} \text { Defined for } f \in C_{0}^{\infty}\left(\mathbb{R}^{n}\right) \text {, by } \widehat{f}(\xi)}:=\int_{\mathbb{R}^{n}} e^{-2 \pi i x \cdot \xi} f(x) d x$.
} 
SU(2) were investigated by Coifman and Weiss in their classical works [17, 18]. Subelliptic Spectral multipliers for $L^{p}(\mathrm{SU}(2))$ were also considered in Cowling and Sikora [14]. Later on, criteria for the $L^{p}$-boundedness of Fourier multipliers for arbitrary compact Lie groups $G$ were given in [44], with a generalisation in [10, Section 5] to $L^{p}$-subelliptic Fourier multipliers.

One of the notable questions when studying the qualitative properties for multipliers on Lie groups is to endow (the spaces of discrete functions on) the unitary dual with a difference structure. So, fixing the unitary dual $\widehat{G}$ of an arbitrary compact Lie group $G$, when generalising the Marcinkiewicz condition on a Fourier multiplier $A$ on $G$, associated to a sequence (called the symbol of $A) \sigma:=\{\sigma(\xi)\}_{[\xi] \in \widehat{G}}$,

$$
A f(x) \equiv T_{\sigma} f(x):=\sum_{[\xi] \in \widehat{G}} d_{\xi} \operatorname{Tr}[\xi(x) \sigma(\xi) \widehat{f}(\xi)], \quad f \in C^{\infty}(G),
$$

the following questions arise:

(Q1): how to define the difference operators $\Delta^{\alpha}$ on $\widehat{G}$, in such a way that they generalise the usual notion of difference operators on $\mathbb{Z} \cong \widehat{\mathbb{T}}$ ?

(Q2): which is the required order for the differences operators applied to $\sigma$ in order that $A$ admits a bounded extension on $L^{p}(G)$ ?

We note that (Q1) was satisfactorily solved in [44] by introducing a family of difference operators $\Delta^{\alpha}:=\mathbb{D}_{\xi}^{\alpha}$, (defined in terms of the Fourier transform on $G$, as it was done in [40]) in terms of the unitary representations $\xi: G \rightarrow \operatorname{End}\left(\mathbb{C}^{\ell}\right)^{3}$ of $G$. About (Q2), the following Marcinkiewicz type theorem was proved in [44].

Theorem 1.1 Let us assume that $G$ is a compact Lie group of dimension $n$. Let $\sigma \in \Sigma(\widehat{G})^{4}$ be a symbol satisfying

$$
\left\|\mathbb{D}^{\alpha} \sigma(\xi)\right\|_{\text {op }} \leqslant C_{\alpha}\langle\xi\rangle^{-|\alpha|},|\alpha| \leqslant \varkappa:=\left[\frac{n}{2}\right]+1 .
$$

Then $A \equiv T_{\sigma}$ is of weak type $(1,1)$ and bounded on $L^{p}(G)$ for all $1<p<\infty$. Moreover,

$$
\|A\|_{\mathscr{B}\left(L^{p}(G)\right)},\|A\|_{\mathscr{B}\left(L^{1}(G), L^{1, \infty}(G)\right)} \lesssim \max \left\{C_{\alpha}:|\alpha| \leq \varkappa\right\} .
$$

Remark 1.2 In the symbol condition (1.7),

$$
\operatorname{Spect}\left(\left(1+\mathcal{L}_{G}\right)^{\frac{1}{2}}\right):=\{\langle\xi\rangle:[\xi] \in \widehat{G}\},
$$

is the system of eigenvalues of the Bessel potential operator $\left(1+\mathcal{L}_{G}\right)^{\frac{1}{2}}$ associated to the Laplacian on $G$, which can be defined as follows. Taking an arbitrary orthonormal basis

\footnotetext{
${ }^{2}$ Here, $[\xi]$ denotes the equivalence class of a unitary, irreducible and continuous representation $\xi: G \rightarrow \operatorname{Hom}\left(\mathbb{C}^{d_{\xi}}\right)$ on $G, \quad d_{\xi}$ is the dimension of its representation space, $\sigma(\xi) \in \operatorname{Hom}\left(\mathbb{C}^{d_{\xi}}\right)$, and $\hat{f}(\xi):=\int_{G} f(x) \xi(x)^{*} d x$, is the Fourier transform on the group $G$, of $f \in C^{\infty}(G)$ at $[\xi]$. For instance, in the case of the torus $G=\mathbb{T}^{n}, \xi(x):=e^{i 2 \pi x \cdot \xi}, x \in \mathbb{U}^{n}, d_{\xi} \equiv 1$, and so $\widehat{G} \cong \mathbb{Z}^{n}$.

${ }^{3}$ We will always write $d_{\xi}:=\ell$ for the dimension of the representation space $\mathbb{C}^{\ell}$. Also, $I_{\ell}$ is the identity matrix of size $\ell \times \ell$.

${ }^{4} \Sigma(\widehat{G})$ is the space of functions $\sigma: \widehat{G} \rightarrow \cup_{\ell \in \mathbb{N}_{0}}\left(\operatorname{End}\left(\mathbb{C}^{\ell}\right)\right)$.
} 
$X_{\mathfrak{g}}:=\left\{X_{1}, \cdots, X_{n}\right\}$ of the Lie algebra $\mathfrak{g}$ of $G$, with respect to the Killing form on $\mathfrak{g}$, $\mathcal{L}_{G}:=-\sum_{i=1}^{n} X_{i}^{2}$. We refer the reader to Remark 2.1 for details about the definition of the difference operators $\mathbb{D}^{\alpha}=\mathbb{D}_{1}^{\alpha_{1}} \cdots \mathbb{D}_{n}^{\alpha_{n}}$. They are compositions of differences operators $\mathbb{D}_{j}$ of first order associated to the entries of the matrix-function $\xi_{0}(\cdot)-I_{d_{\xi_{0}}}$ for any choice of a unitary representation in every equivalence class $\left[\xi_{0}\right] \in \widehat{G}$.

Remark 1.3 Theorem 1.1 was proved by using the Hörmander-Mihlin Theorem in [44, Page 630], see also Theorem 2.6.

Let us note that for graded Lie groups (e.g. the Heisenberg group, any stratified group and a wide class of nilpotent Lie groups where Rockland operators exist, see [26] for details) the Hörmander-Mihlin and the Marcinkiewicz conditions for $L^{p}$, TriebelLizorkin and Hardy spaces have been investigated in [8, 11, 12, 25].

In this work we investigate the Marcinkiewicz condition for multipliers on TriebelLizorkin spaces $F_{p, q}^{r}(G)$ on $G$, extending in Theorem 1.4 to the case of compact Lie groups, the estimate of Seeger [47] for multipliers in Triebel-Lizorkin spaces $F_{p, q}^{r}\left(\mathbb{R}^{n}\right)$ on $\mathbb{R}^{n}$. In order to present our main result, let us define the Triebel-Lizorkin spaces $F_{p, q}^{r}(G)$, as they were introduced by the second author, Nursultanov and Tikhonov in [38]. So, let us fix $\eta \in C_{0}^{\infty}\left(\mathbb{R}^{+},[0,1]\right), \eta \neq 0$, so that $\operatorname{supp}(\eta) \subset[1 / 2,2]$, and such that

$$
\sum_{j \in \mathbb{Z}} \eta\left(2^{-j} \lambda\right)=1, \lambda>0
$$

Fixing $\psi_{0}(\lambda):=\sum_{j=-\infty}^{0} \eta_{j}(\lambda)$, and for $j \geq 1, \psi_{j}(\lambda):=\eta\left(2^{-j} \lambda\right)$, we have

$$
\sum_{\ell=0}^{\infty} \psi_{\ell}(\lambda)=1, \lambda>0,
$$

and one can define the family of operators $\psi_{j}(\mathcal{B})$ using the functional calculus of the elliptic Bessel potential $\mathcal{B}:=\left(1+\mathcal{L}_{G}\right)^{\frac{1}{2}}$. Then, for $0<q<\infty$, and $1<p<\infty$, the Triebel-Lizorkin space $F_{p, q}^{r}(G)$ consists of the distributions $f \in \mathscr{D}^{\prime}(G)$ such that

$$
\|f\|_{F_{p, q}^{r}(G)}:=\left\|\left(\sum_{\ell=0}^{\infty} 2^{\ell r q}\left|\psi_{\ell}(\mathcal{B}) f\right|^{q}\right)^{\frac{1}{q}}\right\|_{L^{p}(G)}<\infty .
$$

The weak- $F_{1, q}^{r}(G)$ space is defined by the distributions $f \in \mathscr{D}^{\prime}(G)$ such that

$$
\|f\|_{\text {weak }-F_{1, q}^{r}(G)}:=\sup _{t>0} t\left|\left\{x \in G:\left(\sum_{\ell=0}^{\infty} 2^{\ell r q}\left|\psi_{\ell}(\mathcal{B}) f(x)\right|^{q}\right)^{\frac{1}{q}}>t\right\}\right|<\infty .
$$

Above, for a measurable subset $A \subset G,|A|$ denotes its Haar measure. The main results of this work are Theorem 1.4 below and the Hörmander-Mihlin Theorem 3.1.

Theorem 1.4 Let us assume that $G$ is a compact Lie group of dimension $n$. Let $\sigma \in \Sigma(\widehat{G})$ be a symbol satisfying 


$$
\left\|\mathbb{D}^{\alpha} \sigma(\xi)\right\|_{\mathrm{op}} \leqslant C_{\alpha}\langle\xi\rangle^{-|\alpha|},|\alpha| \leqslant \varkappa:=\left[\frac{n}{2}\right]+1 .
$$

Then $A \equiv T_{\sigma}$ extends to a bounded operator from $F_{p, q}^{r}(G)$ into $F_{p, q}^{r}(G)$ for all $1<p, q<\infty$, and all $r \in \mathbb{R}$. For $p=1, A$ admits a bounded extension from $\stackrel{p}{F}_{1, q}^{r}(G)$ into weak- $F_{1, q}^{r}(G)$. Moreover

$$
\|A\|_{\mathscr{B}\left(F_{p, q}^{r}(G)\right)},\|A\|_{\mathscr{B}\left(F_{1, q}^{r}(G), \text { weak- } F_{1, q}^{r}(G)\right)} \lesssim \max \left\{C_{\alpha}:|\alpha| \leq \varkappa\right\} .
$$

Remark 1.5 The Marcinkiewicz Theorem 1.4 will be deduced, from the Hörmander-Mihlin Theorem 3.1 for Triebel-Lizorkin spaces. In particular, in view of the Littlewood-Paley theorem (see Furioli, Melzi and Veneruso [29]), we have $L^{p}(G)=F_{p, 2}^{0}(G)$ for $1<p<\infty$, so that we recover the $L^{p}$-bound in Theorem 1.1. Because we are not assuming that the Fourier multipliers are defined by the spectral calculus, Theorem 3.1 extends the main theorem in Weiss [53] and also the historical 1939's result due to Marcinkiwicz [35] (see Remark 4.7 and Corollary 4.8). As before, we refer the reader to Remark 2.1 for details about the definition of the difference operators $\mathbb{D}^{\alpha}=\mathbb{D}_{1}^{\alpha_{1}} \cdots \mathbb{D}_{n}^{\alpha_{n}}$.

\section{Preliminaries}

\subsection{The unitary dual and the Fourier transform}

First, let us record the notion of the unitary dual $\widehat{G}$ of a compact Lie group $G$. So, let us assume that $\xi$ is a continuous, unitary and irreducible representation of $G$, this means that,

- $\xi \in \operatorname{Hom}\left(G, \mathrm{U}\left(H_{\xi}\right)\right)$, for some finite-dimensional vector space $H_{\xi} \cong \mathbb{C}^{d_{\xi}}$, i.e. $\xi(x y)=\xi(x) \xi(y)$ and for the adjoint of $\xi(x), \xi(x)^{*}=\xi\left(x^{-1}\right)$, for every $x, y \in G$.

- The map $(x, v) \mapsto \xi(x) v$, from $G \times H_{\xi}$ into $H_{\xi}$ is continuous.

- For every $x \in G$, and $W_{\xi} \subset H_{\xi}$, if $\xi(x) W_{\xi} \subset W_{\xi}$, then $W_{\xi}=H_{\xi}$ or $W_{\xi}=\emptyset$.

Let $\operatorname{Rep}(G)$ be the set of unitary, continuous and irreducible representations of $G$. The relation,

$$
\xi_{1} \sim \xi_{2} \text { if and only if, there exists } A \in \operatorname{End}\left(H_{\xi_{1}}, H_{\xi_{2}}\right) \text {, such that } A \xi_{1}(x) A^{-1}=\xi_{2}(x),
$$

for every $x \in G$, is an equivalence relation and the unitary dual of $G$, denoted by $\widehat{G}$ is defined via

$$
\widehat{G}:=\operatorname{Rep}(G) / \sim .
$$

By a suitable changes of basis, we always can assume that every $\xi$ is matrix-valued and that $H_{\xi}=\mathbb{C}^{d_{\xi}}$. If a representation $\xi$ is unitary, then

$$
\xi(G):=\{\xi(x): x \in G\}
$$

is a subgroup (of the group of matrices $\mathbb{C}^{d_{\xi} \times d_{\xi}}$ ) which is isomorphic to the original group $G$. Thus the homomorphism $\xi$ allows us to represent the compact Lie group $G$ as a group of matrices. This is the motivation for the term 'representation'. Here, as usually, 


$$
\widehat{f}(\xi) \equiv(\mathscr{F} f)(\xi):=\int_{G} f(x) \xi(x)^{*} d x \in \mathbb{C}^{d_{\xi} \times d_{\xi}}, \quad[\xi] \in \widehat{G},
$$

is the matrix-valued Fourier transform of $f \in C_{0}^{\infty}(G)$ at $\xi=\left(\xi_{i j}\right)_{i, j=1}^{d_{\xi}}$.

\subsection{Difference operators}

Difference operators on compact Lie groups were introduced in [40] to endow the unitary dual of a compact Lie group with a difference structure. In terms of them, Hörmander classes of pseudo-differential operators on a compact Lie group can be characterised, see [41-43]. Same as in [44], where differences operators were used to study $L^{p}$-multipliers we will extend that analysis to the case of Triebel-Lizorkin spaces (see [37, 38] for instance).

We will denote by $\Sigma(\widehat{G})$ the space of matrix-valued functions,

$$
\Sigma(\widehat{G}):=\left\{\sigma \in \mathscr{F}(\mathscr{D}(G))=: \mathscr{D}^{\prime}(\widehat{G}) \mid \sigma: \widehat{G} \rightarrow \cup_{[\xi] \in \widehat{G}} \mathbb{C}^{d_{\xi} \times d_{\xi}}\right\} .
$$

By following [44], a difference operator $Q_{\xi}$ of order $k$, can be applied to a symbol $\sigma=\widehat{f} \in \mathscr{D}(\widehat{G})$, via

$$
Q_{\xi} \sigma(\xi)=\widehat{q f}(\xi),[\xi] \in \widehat{G}
$$

where $Q_{\xi}$ is associated with a smooth function $q$ vanishing of order $k$ at the identity $e=e_{G}$. We will denote by $\operatorname{diff}^{k}(\widehat{G})$ the set of all difference operators of order $k$. For a fixed smooth function $q$, the associated difference operator will be denoted by $\Delta_{q}:=Q_{\xi}$. We will choose an admissible collection of difference operators (see e.g. [44]),

$$
\Delta_{\xi}^{\alpha}:=\Delta_{q_{(1)}}^{\alpha_{1}} \cdots \Delta_{q_{(i)}}^{\alpha_{i}}, \alpha=\left(\alpha_{j}\right)_{1 \leqslant j \leqslant i},
$$

where

$$
\operatorname{rank}\left\{\nabla q_{(j)}(e): 1 \leqslant j \leqslant i\right\}=\operatorname{dim}(G) \text {, and } \Delta_{q_{(j)}} \in \operatorname{diff}^{1}(\widehat{G}) .
$$

We say that this admissible collection is strongly admissible if

$$
\bigcap_{j=1}^{i}\left\{x \in G: q_{(j)}(x)=0\right\}=\left\{e_{G}\right\} .
$$

Remark 2.1 A special type of difference operators can be defined by using the unitary representations of $G$. Indeed, if $\xi_{0}$ is a fixed irreducible and unitary representation of $G$, consider the matrix

$$
\xi_{0}(g)-I_{d_{\xi_{0}}}=\left[\xi_{0}(g)_{i j}-\delta_{i j}\right]_{i, j=1}^{d_{\xi}}, \quad g \in G .
$$

Then, we associated to the function $q_{i j}(g):=\xi_{0}(g)_{i j}-\delta_{i j}, \quad g \in G$, a difference operator via

$$
\mathbb{D}_{\xi_{0}, i, j}:=\mathscr{F}\left(\xi_{0}(g)_{i j}-\delta_{i j}\right) \mathscr{F}^{1} .
$$


If the representation is fixed we omit the index $\xi_{0}$ so that, from a sequence $\mathbb{D}_{1}=\mathbb{D}_{\xi_{0}, j_{1}, i_{1}}, \cdots, \mathbb{D}_{n}=\mathbb{D}_{\xi_{0}, j_{n}, i_{n}}$ of operators of this type we define $\mathbb{D}^{\alpha}=\mathbb{D}_{1}^{\alpha_{1}} \cdots \mathbb{D}_{n}^{\alpha_{n}}$, where $\alpha \in \mathbb{N}^{n}$.

Remark 2.2 (Leibniz rule for difference operators) The difference structure on the unitary dual $\widehat{G}$, induced by the difference operators acting on the momentum variable $[\xi] \in \widehat{G}$, implies the following Leibniz rule

$$
\mathbb{D}^{\alpha}\left(a_{1} a_{2}\right)\left(x_{0}, \xi\right)=\sum_{|\gamma|,|\varepsilon| \leqslant|\alpha| \leqslant|\gamma|+|\varepsilon|} C_{\varepsilon, \gamma}\left(\mathbb{D}^{\gamma} a_{1}\right)\left(x_{0}, \xi\right)\left(\mathbb{D}^{\varepsilon} a_{2}\right)\left(x_{0}, \xi\right), \quad\left(x_{0},[\xi]\right) \in G \times \widehat{G},
$$

for $a_{1}, a_{2} \in C^{\infty}\left(G, \mathscr{D}^{\prime}(\widehat{G})\right)$. For details we refer the reader to [44, Page 631].

Remark 2.3 (Difference operators of fractional order and Sobolev spaces on the unitary dual) In the spirit of the Sobolev spaces on the unitary dual of a graded Lie group [24], Sobolev spaces also can be defined for the unitary dual of a compact Lie group. They can be defined as follows: Let $\Delta_{q_{1}}$, be a difference operator of first order associated to a smooth and non-negative function $q_{1} \geq 0$. For $s \in \mathbb{R}$, the Sobolev space $\dot{L}_{s}^{2}(\widehat{G})$ consists of all distributions $\sigma=\widehat{f} \in \mathscr{D}(G)$ such that

$$
\|\sigma\|_{\dot{L}_{s}^{2}(\widehat{G})}:=\|f\|_{L^{2}\left(G, q_{1}^{2 s}\right)}=\left\|q_{1}^{s} f\right\|_{L^{2}(G)}<\infty .
$$

For $s \in \mathbb{N}$, observe that,

$$
\|\sigma\|_{\dot{L}_{s}^{2}(\widehat{G})} \asymp \max _{|\alpha|=s}\left\|\Delta_{\xi}^{\alpha} \sigma\right\|_{L^{2}(\widehat{G})} \asymp \max _{|\alpha|=s}\left\|\mathbb{D}_{\xi}^{\alpha} \sigma\right\|_{L^{2}(\widehat{G})} .
$$

So, for every $s \in \mathbb{R}$, the difference operator $\Delta_{q_{1}}^{s}:=\Delta_{q_{1}^{s}}$ of fractional order $s$, can be defined in terms of the Fourier transform, via:

$$
\Delta_{q_{1}}^{s} \widehat{f}=\widehat{q_{1}^{s} f}, \quad f \in \mathscr{D}^{\prime}(G) .
$$

So, we have $\|\sigma\|_{\dot{L}_{s}^{2}(\widehat{G})}:=\left\|\Delta_{q_{1}}^{s} \widehat{f}\right\|_{L^{2}(\widehat{G})}$. We will denote

$$
\operatorname{diff}^{s}(\widehat{G}):=\left\{\Delta_{q_{1}^{s}}: \Delta_{q_{1}} \in \operatorname{diff}^{1}(\widehat{G})\right\}
$$

\subsection{Calderón-Zygmund type estimates for multipliers}

In order to provide $L^{p}$-estimates for multipliers in the subelliptic context, we will use the techniques developed by the second author and J. Wirth in [44], where a special case (compatible with the notion of difference operators and the difference structure that they provide for the unitary dual) of a statement of Coifman and de Guzmán ([16], Theorem 2) was established. We record it as follows (see [44, p. 630]).

Theorem 2.4 (Hörmander-Mihlin Theorem for $L^{p}(G)$ ) Assume that $A: L^{2}(G) \rightarrow L^{2}(G)$ is a left-invariant operator on $G$ satisfying 


$$
\left\|A \psi_{r^{-1}}\right\|_{L^{2}\left(G, \rho(x)^{n(1+\varepsilon)} d x\right)}:=\left(\int_{G}\left|A \psi_{r^{-1}}(x)\right|^{2} \rho(x)^{n(1+\varepsilon)} d x\right)^{\frac{1}{2}} \lesssim C r^{-\frac{\varepsilon}{2}},
$$

for some $\varepsilon>0$, uniformly in $r>0$. Then $A$ is of weak type $(1,1)$ and bounded on $L^{p}(G)$, for all $1<p<\infty$.

The family $\left\{\psi_{r}\right\}_{r>0}$ that appears in Theorem 2.4 is defined by

$$
\psi_{r}:=\phi_{r}-\phi_{r / 2}
$$

where the functions in the net $\left\{\phi_{r}\right\}_{r>0}$, satisfy, among other things, the following properties (see [44, Lemma 3.3]):

- $\int \phi_{r}(x) d x=1$,

- $\int_{G}^{G} \phi_{r}^{2}(x) d x=O\left(\frac{1}{r}\right)$

- $\phi_{r} * \phi_{s}=\phi_{s} * \phi_{r}, r, s>0$.

The function $\rho: x \mapsto \rho(x)$, appearing in (2.8), is a suitable pseudo-distance defined on $G$. If $G$ is semi-simple (this means that the centre of $G, Z(G)$ is trivial), it is defined by

$$
\rho(x)^{2}:=\operatorname{dim}(G)-\operatorname{Tr}(\operatorname{Ad}(x))=\sum_{\xi \in \Delta_{0}}\left(d_{\xi}-\operatorname{Tr}(\xi(x))\right), x \in G,
$$

where Ad : $G \rightarrow \mathrm{U}(\mathfrak{g})$, and $\Delta_{0}$ is the system of positive roots. It can be decomposed into irreducible representations as,

$$
\operatorname{Ad}=\left[\operatorname{rank}(G) e_{\hat{G}}\right] \oplus\left(\bigoplus_{\xi \in \Delta_{0}} \xi\right)
$$

where $e_{\widehat{G}}$ is the trivial representation. With the consideration on the centre $Z(G)=\left\{e_{G}\right\}$, it can be shown (see Lemma 3.1 of [44]) that

- $\rho^{2}(x) \geqslant 0$ and $\rho(x)=0$ if and only if $x=e_{G}$.

- $\Delta_{\rho^{2}} \in \operatorname{diff}^{2}(\widehat{G})$.

If $G$ is not semi-simple, we refer the reader to [44, Remark 3.2] for the modifications in the definition of $\rho$, in this particular case. The construction of the functions $\phi_{r}$, is as follows. By choosing $\tilde{\phi} \in C_{0}^{\infty}(\mathbb{R})$ such that $\tilde{\phi} \geq 0, \tilde{\phi}(0) \equiv 1$, and $\partial_{t}^{\ell} \tilde{\phi}(0)=0$, for $\ell \geq 1$, we define

$$
\psi_{r}(g):=c_{r} \tilde{\psi}\left(r^{-\frac{1}{n}} \rho(g)\right), \quad \int_{G} \psi_{r}(g) d g=1,
$$

with the normalisation condition used to define $c_{r}$. An equivalent statement to the Hörmander-Mihlin Theorem 2.4 will be given in terms of the family of functions $\varphi_{r^{-1}} \equiv \psi_{r^{-n}}, r>0$, (see Theorem 2.6) that provide a continuous dyadic decomposition. 


\section{$2.4 L^{p}(G)$-boundedness of Fourier multipliers}

In this subsection we recall the Hörmander-Mihlin theorem and the MarcinkiewiczMihlin theorem for $L^{p}$-multipliers on compact Lie groups [44].

Remark 2.5 Let $A$ be a Fourier multiplier with symbol $\sigma$. Let us observe that that Theorem 2.4 can be re-written in terms of the Sobolev spaces $\dot{L}_{s}^{2}(\widehat{G})$ on the unitary dual, showing that, (2.8) is indeed, an analogue on compact Lie groups of the Hörmander-Mihlin theorem in [34]. So, with the notation in Theorem 2.4, and making use of the Plancherel theorem, we have

$$
\begin{aligned}
\left\|A \psi_{r}\right\|_{L^{2}\left(G, \rho(x)^{n(1+\varepsilon) d x}\right)}^{2} & :=\int_{G}\left|A \psi_{r}(x)\right|^{2} \rho(x)^{n(1+\varepsilon)} d x \\
& =\left\|\Delta_{\rho(x)}^{n(1+\varepsilon) / 2} \sigma(\xi) \widehat{\psi}_{r}(\xi)\right\|_{L^{2}(\widehat{G})}^{2} \\
& :=\left\|\sigma(\xi) \widehat{\psi}_{r}(\xi)\right\|_{L_{s}^{2}(\widehat{G})}^{2},
\end{aligned}
$$

where $s:=n(1+\varepsilon) / 2>n / 2$. Observing that $\frac{\varepsilon}{2}=\frac{1}{n}\left(s-\frac{n}{2}\right)$, and that $A: L^{2}(G) \rightarrow L^{2}(G)$ is bounded if and only if, $\|\sigma\|_{L^{\infty}(\widehat{G})}:=\sup _{[\xi] \in \widehat{G}}\|\sigma(\xi)\|_{\mathrm{op}}<\infty$, Theorem 2.4 is equivalent to the following theorem. We set

$$
\varphi_{r^{-1}} \equiv \psi_{r^{-n}}:=\phi_{r^{-n}}-\phi_{r^{-n} / 2}, \quad r>0,
$$

for the continuous dyadic approximation of the identity in (2.9).

Theorem 2.6 (Hörmander-Mihlin Theorem for $L^{p}(G)$ ) Let $G$ be a compact Lie group and let $s>\frac{n}{2}$. Let $\sigma \in \Sigma(\widehat{G})$ be a symbol satisfying

$$
\|\sigma\|_{l \cdot u, L_{s}^{2}(\widehat{G})}:=\|\sigma\|_{L^{\infty}(\widehat{G})}+\sup _{r>0} r^{\left(s-\frac{n}{2}\right)}\left\|\sigma \cdot \widehat{\varphi}_{r^{-1}}\right\|_{\dot{L}_{s}^{2}(\widehat{G})}<\infty .
$$

Then $A \equiv T_{\sigma}$ is of weak type $(1,1)$ and bounded on $L^{p}(G)$ for all $1<p<\infty$.

We present Theorem 1.1 in the following form.

Theorem 2.7 (Marcinkiewicz-Mihlin Theorem) Let $G$ be a compact Lie group and let $\varkappa \in 2 \mathbb{N}$ be such that $\varkappa>\frac{n}{2}$. Let $\sigma \in \Sigma(\widehat{G})$ be a symbol satisfying

$$
\left\|\mathbb{D}^{\alpha} \sigma(\xi)\right\|_{\mathrm{op}} \leqslant C_{\alpha}\langle\xi\rangle^{-|\alpha|},|\alpha| \leqslant \varkappa .
$$

Then $A \equiv T_{\sigma}$ is of weak type $(1,1)$ and bounded on $L^{p}(G)$ for all $1<p<\infty$.

Remark 2.8 Several properties for the Sobolev spaces $L_{s}^{2}(\widehat{G})$ on the unitary dual were established in [23]. In particular, the Hörmander-Mihlin Theorem 2.6 was re-obtained, by observing that the $L^{p}(G)$-boundedness and the weak $(1,1)$ type of a Fourier multiplier, also can be obtained if one verifies the following condition:

$$
\|\sigma\|_{l . u ., L_{s}^{2}(\widehat{G})}^{\prime}:=\|\sigma\|_{L^{\infty}(\widehat{G})}+\sup _{r>0} r^{\left(s-\frac{n}{2}\right)}\left\|\sigma \cdot \eta\left(r^{-1}\langle\xi\rangle\right)\right\|_{\dot{L}_{s}^{2}(\widehat{G})}<\infty,
$$


for any $\eta \neq 0, \eta \in C_{0}^{\infty}\left(\mathbb{R}_{0}^{+}\right)$, and $s>n / 2$. Both Hörmander-Mihlin conditions $((2.12)$ or (2.13)) allow us to write Theorem 2.7 in the form of Theorem 1.1.

Remark 2.9 The boundedness of pseudo-differential operators on compact Lie groups, including oscillating Fourier multipliers, on $L^{p}$, subelliptic Sobolev and Besov spaces can be found in $[8,15]$ and $[5,6,12]$. The boundedness of Fourier multipliers on $L^{p}$-spaces, Triebel-Lizorkin spaces and Hardy spaces on graded Lie groups can be found in $[8,25]$ and [33], respectively.

\subsection{Triebel-Lizorkin spaces on compact Lie groups}

In this section, Triebel-Lizorkin spaces on compact Lie groups are introduced. This family of spaces was introduced by the second author, Nursultanov and Tikhonov in [38] by using a dyadic partition of the spectral resolution of the Bessel potential $\mathcal{B}=\left(1+\mathcal{L}_{G}\right)^{\frac{1}{2}}$.

As in the introduction, let us fix $\eta \in C_{0}^{\infty}\left(\mathbb{R}^{+},[0,1]\right), \eta \neq 0$, so that $\operatorname{supp}(\eta) \subset[1 / 2,2]$, and such that

$$
\sum_{j \in \mathbb{Z}} \eta\left(2^{-j} \lambda\right)=1, \lambda>0
$$

Fixing $\psi_{0}(\lambda):=\sum_{j=-\infty}^{0} \eta_{j}(\lambda)$, and for $j \geq 1, \psi_{j}(\lambda):=\eta\left(2^{-j} \lambda\right)$, we have

$$
\sum_{\ell=0}^{\infty} \psi_{\ell}(\lambda)=1, \lambda>0 .
$$

Let us define the family of operators $\psi_{j}(\mathcal{B})$ using the functional calculus. Then, for $0<q<\infty$, and $1<p<\infty$, the Triebel-Lizorkin space $F_{p, q}^{r}(G)$ consists of the distributions $f \in \mathscr{D}^{\prime}(G)$ such that

$$
\|f\|_{F_{p, q}^{r}(G)}:=\left\|\left(\sum_{\ell=0}^{\infty} 2^{\ell r q}\left|\psi_{\ell}(\mathcal{B}) f\right|^{q}\right)^{\frac{1}{q}}\right\|_{L^{p}(G)}<\infty,
$$

and for $p=1$, the weak- $F_{1, q}^{r}(G)$ space is defined by the distributions $f \in \mathscr{D}^{\prime}(G)$ such that

$$
\|f\|_{\text {weak }-F_{1, q}^{r}(G)}:=\sup _{t>0} t\left|\left\{x \in G:\left(\sum_{\ell=0}^{\infty} 2^{\ell r q}\left|\psi_{\ell}(\mathcal{B}) f(x)\right|^{q}\right)^{\frac{1}{q}}>t\right\}\right|<\infty .
$$

In the following theorem we present some embedding properties for Triebel-Lizorkin spaces. For the proof of Theorem 2.10 and for a consistent investigation of Triebel-Lizorkin spaces on compact Lie groups, we refer the reader to [38].

Theorem 2.10 Let G be a compact Lie group. Then we have the following properties:

(1) $F_{p, q_{1}}^{r+\varepsilon, \mathcal{L}}(G) \hookrightarrow F_{p, q_{1}}^{r, \mathcal{L}}(G) \hookrightarrow F_{p, q_{2}}^{r, \mathcal{L}}(G) \hookrightarrow F_{p, \infty}^{r, \mathcal{L}}(G), \varepsilon>0,0 \leq p \leq \infty, 0 \leq q_{1} \leq q_{2} \leq \infty$.

(2) $F_{p, q_{1}}^{r+\varepsilon, \mathcal{L}}(G) \hookrightarrow F_{p, q_{2}}^{r, \mathcal{L}}(G), \varepsilon>0,0 \leq p \leq \infty, 1 \leq q_{2}<q_{1}<\infty$. 
(3) $\quad F_{p, 2}^{r}(G)=L_{r}^{p}(G)$ for all $r \in \mathbb{R}$, and all $1<p<\infty$, where $L_{r}^{p}(G)$ are the standard Sobolev spaces on $G$.

Remark 2.11 Let us observe that (3) in Theorem 2.10 is a consequence of the LittlewoodPaley theorem. For details we refer the reader to Furioli, Melzi and Veneruso [29].

\section{Proof of the Marcinkiewicz theorem 1.4}

Let us fix $\eta \in C_{0}^{\infty}\left(\mathbb{R}^{+},[0,1]\right), \eta \neq 0$, so that $\operatorname{supp}(\eta) \subset[1 / 2,2]$, and such that

$$
\sum_{j \in \mathbb{Z}} \eta\left(2^{-j} \lambda\right)=1, \lambda>0
$$

By defining $\psi_{0}(\lambda):=\sum_{j=-\infty}^{0} \eta_{j}(\lambda)$, and for $j \geq 1, \psi_{j}(\lambda):=\eta\left(2^{-j} \lambda\right)$, we obviously have

$$
\sum_{\ell=0}^{\infty} \psi_{\ell}(\lambda)=1, \lambda>0 .
$$

First, let us deduce the proof of Theorem 1.4 from the following result, which will be proved in Sect. 4. The distribution $\vartheta_{t}:=\eta(t \mathcal{B}) \delta$ denotes the right-convolution kernel of the operator $\eta(t \mathcal{B})$.

Theorem 3.1 (Hörmander-Mihlin Theorem for $F_{p, q}^{r}(G)$ ) Let us consider a Fourier multiplier $A: C^{\infty}(G) \rightarrow L^{2}(G)$ satisfying the symbol condition

$$
\|\sigma\|_{l . u ., L_{s}^{2}(\widehat{G})}^{\prime}:=\|\sigma\|_{L^{\infty}(\widehat{G})}+\sup _{r>0} r^{\left(s-\frac{n}{2}\right)}\left\|\sigma \cdot \eta\left(r^{-1}\langle\xi\rangle\right)\right\|_{\dot{L}_{s}^{2}(\widehat{G})}<\infty,
$$

with $s>\frac{n}{2}$. Then $A \equiv T_{\sigma}$ extends to a bounded operator from $F_{p, q}^{r}(G)$ into $F_{p, q}^{r}(G)$ for all $1<p, q<\infty$, and all $r \in \mathbb{R}$. For $p=1, A$ admits a bounded extension from $F_{1, q}^{r}(G)$ into weak- $F_{1, q}^{r}(G)$. Moreover

$$
\|A\|_{\mathscr{B}\left(F_{p, q}^{r}(G)\right)},\|A\|_{\mathscr{B}\left(F_{1, q}^{r}(G), \text { weak- } F_{1, q}^{r}(G)\right)} \lesssim\|\sigma\|_{l . u ., L_{s}^{2}(\widehat{G})}^{\prime} .
$$

Remark 3.2 Note that the Fourier transform of the distribution $\vartheta_{t}:=\eta(t \mathcal{B}) \delta$ is given by

$$
\widehat{\vartheta}_{t}(\xi):=\eta(t\langle\xi\rangle) I_{d_{\xi}},[\xi] \in \widehat{G},
$$

and that $A: L^{2}(G) \rightarrow L^{2}(G)$ is bounded if and only if $\|\sigma\|_{L^{\infty}(\widehat{G})}<\infty$. Let $\Delta_{q_{1}^{s}} \in \operatorname{diff}^{s}(\widehat{G})$ be a fractional difference operator of order $s:=\frac{n+\varepsilon}{2}$, with $\varepsilon>0$. If $A: L^{2}(G) \rightarrow L^{2}(G)$ is bounded and it satisfies the (Coifman-Weiss type) condition

$$
\left\|A \vartheta_{r^{-1}}\right\|_{L^{2}\left(G, q_{1}(x)^{\varepsilon+n} d x\right)}:=\left(\int_{G}\left|A \vartheta_{r^{-1}}(x)\right|^{2} q_{1}(x)^{\varepsilon+n} d x\right)^{\frac{1}{2}} \lesssim_{\varepsilon} C r^{-\frac{1}{2} \varepsilon},
$$

uniformly in $r>0$, the Plancherel Theorem makes the hypothesis in Theorem 3.1 equivalent to (3.6). Moreover, (3.4) is equivalent to 
$\|A\|_{\mathscr{B}\left(F_{p, q}^{r}(G)\right)},\|A\|_{\mathscr{B}\left(F_{1, q}^{r}(G), \text { weak- }-F_{1, q}^{r}(G)\right)} \lesssim\|\sigma\|_{L^{\infty}(\widehat{G})}+\sup _{r>0} r^{s-\frac{n}{2}}\left\|A \vartheta_{r^{-1}}\right\|_{L^{2}\left(G, q_{1}(x)^{2 s} d x\right)}$,

in view of (3.5).

Four our further analysis we will use the following auxiliary estimate.

Lemma 3.3 Let A be a Fourier multiplier satisfying the hypothesis in Theorem 1.4. Then, the following estimate holds,

$$
\left\|\sigma(\xi) \psi_{\ell}(\langle\xi\rangle)\right\|_{\dot{L}_{2}^{s}(\widehat{G})} \lesssim_{s, \psi} 2^{-\ell\left(s-\frac{n}{2}\right)} \sup _{|\alpha| \leq s} \sup _{[\xi] \in \widehat{G}}\left\|\left(\Delta_{\xi}^{\alpha} \sigma(\xi)\right)\langle\xi\rangle^{|\alpha|}\right\|_{\mathrm{op}}, \quad \ell \in \mathbb{Z},
$$

provided that $s>n / 2, s \in \mathbb{N}$. Moreover, the right-convolution kernel $\kappa_{\ell}$ of $A \psi_{\ell}$, satisfies the uniform estimate in $\ell \in \mathbb{Z}$,

$$
\int_{|x|>4 c|z|}\left|\kappa_{\ell}\left(z^{-1} x\right)-\kappa_{\ell}(x)\right| d x \lesssim 2^{-\ell\left(s-\frac{n}{2}\right)}\|\sigma\|_{l . u, L_{s}^{2}(\widehat{G})}^{\prime} .
$$

Proof That (3.9) is a consequence of (3.8) was proved in [23, Page 38]. So, let us continue with the first part of the lemma. Let us observe that, (in view of Proposition 4.13 in [23]), we have the following norm estimates

$$
\|\sigma \tau\|_{\dot{L}_{s}^{2}(\widehat{G})} \lesssim_{s} \sum_{s_{1}+s_{2}=s}\|\sigma\|_{\dot{L}_{s_{1}}^{\infty}(\widehat{G})}\|\tau\|_{\dot{L}_{s_{2}}^{2}(\widehat{G})}, \quad\|\sigma\|_{\dot{L}_{s_{1}}^{\infty}(\widehat{G})}:=\max _{|\alpha|=s_{1}}\left\|\Delta_{\xi}^{\alpha} \sigma\right\|_{L^{\infty}(\widehat{G})}
$$

in the sense that if the right-hand side is finite then the left-hand side is finite and the inequality holds. So, applying (3.10) to $\tau=\psi_{\ell}(\langle\xi\rangle) I_{d_{\xi}}$, we have

$$
\left\|\sigma \psi_{\ell}(\langle\xi\rangle)\right\|_{\dot{L}_{s}^{2}(\widehat{G})} \lesssim_{s} \sum_{s_{1}+s_{2}=s} \max _{|\alpha|=s_{1}}\left\|\Delta_{\xi}^{\alpha} \sigma\right\|_{L^{\infty}(\widehat{G})}\left\|\psi_{\ell}(\langle\xi\rangle) I_{d_{\xi}}\right\|_{\dot{L}_{s_{2}}^{2}(\widehat{G})} .
$$

Observe that,

$$
\left\|\Delta_{\xi}^{\alpha} \sigma\right\|_{L^{\infty}(\widehat{G})} \leq\left\|\Delta_{\xi}^{\alpha} \sigma\langle\xi\rangle^{|\alpha|}\right\|_{L^{\infty}(\widehat{G})}\left\|\widehat{\mathcal{B}}(\xi)^{-|\alpha|}\right\|_{L^{\infty}(\widehat{G})} \leq\left\|\Delta_{\xi}^{\alpha} \sigma\langle\xi\rangle^{|\alpha|}\right\|_{L^{\infty}(\widehat{G})} .
$$

Also, in view of Lemma 5.4 of [23], for any positive and smooth function $f \in C_{0}^{\infty}(\mathbb{R})$, supported in $[0, a]$ with $a>0$, one has

$$
\left\|f(t\langle\xi\rangle) I_{d_{\xi}}\right\|_{\dot{L}_{s}^{2}(\widehat{G})} \lesssim_{s} t^{s-\frac{n}{2}}\|f\|_{H^{s^{\prime}(\mathbb{R})}}, s^{\prime}>s+1 / 2,0<t \leq 1,
$$

which applied to $f=\psi$ and $t=2^{-\ell}$, gives

$$
\left\|\psi_{\ell}(\langle\xi\rangle) I_{d_{\xi}}\right\|_{\dot{L}_{s_{2}}^{2}(\widehat{G})} \lesssim\left\|\psi_{\ell}(\langle\xi\rangle) I_{d_{\xi}}\right\|_{\dot{L}_{s}^{2}(\widehat{G})} \lesssim_{S} 2^{-\ell\left(s-\frac{n}{2}\right)}\|\psi\|_{H^{s^{\prime}}(\mathbb{R})} .
$$

The analysis above shows that

$$
\left\|\sigma(\xi) \psi_{\ell}(\langle\xi\rangle)\right\|_{\dot{L}_{2}^{s}(\widehat{G})} \lesssim_{s} \sup _{|\alpha| \leq s}\left\|\Delta_{\xi}^{\alpha} \sigma\langle\xi\rangle^{|\alpha|}\right\|_{L^{\infty}(\widehat{G})} 2^{-\ell\left(s-\frac{n}{2}\right)} .
$$

Thus, the proof is complete. 
Proof of Theorem 1.4 In view of Lemma 3.3, we have that

$$
\begin{aligned}
\|\sigma\|_{l \cdot u ., L_{s}^{2}(\widehat{G})}^{\prime} & :=\|\sigma\|_{L^{\infty}(\widehat{G})}+\sup _{r>0} r^{\left(s-\frac{n}{2}\right)}\left\|\sigma \cdot \eta\left(r^{-1}\langle\xi\rangle\right)\right\|_{\dot{L}_{s}^{2}(\widehat{G})} \\
& \lesssim_{S, \psi} \sup _{|\alpha| \leq s} \sup _{[\xi] \in \widehat{G}}\left\|\left(\Delta_{\xi}^{\alpha} \sigma(\xi)\right)\langle\xi\rangle^{|\alpha|}\right\|_{\mathrm{op}} \lesssim \sup _{|\alpha| \leq s} C_{\alpha} .
\end{aligned}
$$

In view of the Hörmander-Mihlin Theorem 3.1 applied to $s:=\left[\frac{n}{2}\right]+1, A \equiv T_{\sigma}$ extends to a bounded operator from $F_{p, q}^{r}(G)$ into $F_{p, q}^{r}(G)$ for all $1<p, q<\infty$, and all $r \in \mathbb{R}$. For $p=1, A$ admits a bounded extension from $F_{1, q}^{r}(G)$ into weak- $F_{1, q}^{r}(G)$.

\section{Proof of the Hörmander-Mihlin theorem 3.1}

Let us define the vector-valued operator $W: L^{2}\left(G, \ell^{2}\left(\mathbb{N}_{0}\right)\right) \rightarrow L^{2}\left(G, \ell^{2}\left(\mathbb{N}_{0}\right)\right)$ by

$$
W\left(\left\{g_{\ell}\right\}_{\ell=0}^{\infty}\right):=\left(\left\{W_{\ell} g_{\ell}\right\}_{\ell=0}^{\infty}\right), W_{\ell}:=A \psi_{\ell}(\mathcal{B}) .
$$

Observe that $W$ is well-defined (bounded from $L^{2}\left(G, \ell^{2}\left(\mathbb{N}_{0}\right)\right)$ into $L^{2}\left(G, \ell^{2}\left(\mathbb{N}_{0}\right)\right)$ ), because $A$ admits a bounded extension on $L^{2}(G)$ and also, in view of the following estimate

$$
\begin{aligned}
\left\|W\left(\left\{g_{\ell}\right\}_{\ell=0}^{\infty}\right)\right\|_{L^{2}\left(G, \ell^{2}\left(\mathbb{N}_{0}\right)\right)}^{2} & :=\int_{G} \sum_{\ell=0}^{\infty}\left|A \psi_{\ell}(\mathcal{B}) g_{\ell}(x)\right|^{2} d x=\sum_{\ell=0}^{\infty} \int_{G}\left|A \psi_{\ell}(\mathcal{B}) g_{\ell}(x)\right|^{2} d x \\
& \leq\|A\|_{\mathscr{B}\left(L^{2}(G)\right)}^{2} \sup _{\ell}\left\|\psi_{\ell}(\mathcal{B})\right\|_{\mathscr{B}\left(L^{2}(G)\right)}^{2} \sum_{\ell=0}^{\infty} \int_{G}\left|g_{\ell}(x)\right|^{2} d x \\
& \leq\|A\|_{\mathscr{B}\left(L^{2}(G)\right)}^{2} \sup _{\ell}\left\|\psi_{\ell}\right\|_{L^{\infty}(\mathbb{R})}^{2} \sum_{\ell=0}^{\infty} \int_{G}\left|g_{\ell}(x)\right|^{2} d x \\
& =\|A\|_{\mathscr{B}\left(L^{2}(G)\right)}^{2}\|\psi\|_{L^{\infty}(\mathbb{R})}^{2} \sum_{\ell=0}^{\infty} \int_{G}\left|g_{\ell}(x)\right|^{2} d x \\
& \lesssim\left\|\left\{g_{\ell}\right\}_{\ell=0}^{\infty}\right\|_{L^{2}\left(G, \ell^{2}\left(\mathbb{N}_{0}\right)\right)}^{2}
\end{aligned}
$$

So, observe that in order to prove Theorem 3.1 it is enough to prove the following two lemmas and the estimate (3.4).

Lemma 4.1 Let $G$ be a compact Lie group and let $1<q<\infty$. Then, $W: L^{q}\left(G, \ell^{q}\left(\mathbb{N}_{0}\right)\right) \rightarrow L^{q}\left(G, \ell^{q}\left(\mathbb{N}_{0}\right)\right)$ admits a bounded extension.

Lemma 4.2 Let $G$ be a compact Lie group and let $1<q<\infty$. Then, $W: L^{1}\left(G, \ell^{q}\left(\mathbb{N}_{0}\right)\right) \rightarrow L^{1, \infty}\left(G, \ell^{q}\left(\mathbb{N}_{0}\right)\right)$ admits a bounded extension.

Corollary 4.3 Let $G$ be a compact Lie group and let $1<p, q<\infty$. Then, $W: L^{p}\left(G, \ell^{q}\left(\mathbb{N}_{0}\right)\right) \rightarrow L^{p}\left(G, \ell^{q}\left(\mathbb{N}_{0}\right)\right)$ admits a bounded extension.

Proof Indeed, by the Marcinkiewicz interpolation, these two lemmas are enough to show that $W: L^{p}\left(G, \ell^{q}\left(\mathbb{N}_{0}\right)\right) \rightarrow L^{p}\left(G, \ell^{q}\left(\mathbb{N}_{0}\right)\right)$ admits a bounded extension for all 
$1<p \leq q<\infty$. The case $1<q \leq p<\infty$ follows from the fact that $L^{p^{\prime}}\left(G, \ell^{q^{\prime}}\left(\mathbb{N}_{0}\right)\right)$ is the dual of $L^{p}\left(G, \ell q\left(\mathbb{N}_{0}\right)\right)$ and also that Lemma 4.1 and Lemma 4.2 hold if we change $A$ by its standard $L^{2}$-adjoint.

Indeed, we clarify this claim in the following remark.

Remark 4.4 (Lemma 4.2 and Corollary 4.3 imply Theorem 3.1) By observing that $(1+\mathcal{B})^{\frac{r}{2}}: F_{p, q}^{r}(G) \rightarrow F_{p, q}^{0}(G)$ and $(1+\mathcal{B})^{-\frac{r}{2}}: F_{p, q}^{0}(G) \rightarrow F_{p, q}^{r}(G)$ are both isomorphisms, it is enough to prove that $A$ admits a bounded extension from $F_{p, q}^{0}(G)$ into $F_{p, q}^{0}(G)$. By defining $\psi_{-1}=\psi_{0}$, from Corollary 4.3 we have that

$$
\begin{aligned}
\|A f\|_{F_{p, q}^{0}(G)} & =\left\|\left(\sum_{l=0}^{\infty}\left|A \psi_{l}(\mathcal{B}) f\right|^{q}\right)^{\frac{1}{q}}\right\|_{L^{p}(G)} \\
& \leq\left\|\left(\sum_{l=0}^{\infty}\left|A \psi_{l}(\mathcal{B})\left[\psi_{l-1}(\mathcal{B})+\psi_{l}(\mathcal{B})+\psi_{l+1}(\mathcal{B})\right] f\right|^{q}\right)^{\frac{1}{q}}\right\|_{L^{p}(G)} \\
& =\left\|W\left(\left\{\left[\psi_{l-1}(\mathcal{B})+\psi_{l}(\mathcal{B})+\psi_{l+1}(\mathcal{B})\right] f\right\}_{l=0}^{\infty}\right)\right\|_{L^{p}\left(\ell^{q}\right)} \\
& \left.\lesssim \|\left\{\left[\psi_{l-1}(\mathcal{B})+\psi_{l}(\mathcal{B})+\psi_{l+1}(\mathcal{B})\right] f\right\}_{l=0}^{\infty}\right) \|_{L^{p}\left(\ell^{q}\right)} \\
& \lesssim\|f\|_{F_{p, q}^{0}(G)} .
\end{aligned}
$$

Also note that from Lemma 4.2, we have

$$
\begin{aligned}
\|A f\|_{\text {weak }-F_{1, q}^{0}(G)} & =\left\|\left(\sum_{l=0}^{\infty}\left|A \psi_{l}(\mathcal{B}) f\right|^{q}\right)^{\frac{1}{q}}\right\|_{L^{1, \infty}(G)} \\
& \leq\left\|\left(\sum_{l=0}^{\infty}\left|A \psi_{l}(\mathcal{B})\left[\psi_{l-1}(\mathcal{B})+\psi_{l}(\mathcal{B})+\psi_{l+1}(\mathcal{B})\right] f\right|^{q}\right)^{\frac{1}{q}}\right\|_{L^{1, \infty}(G)} \\
& =\left\|W\left(\left\{\left[\psi_{l-1}(\mathcal{B})+\psi_{l}(\mathcal{B})+\psi_{l+1}(\mathcal{B})\right] f\right\}_{l=0}^{\infty}\right)\right\|_{L^{1, \infty}(\ell q)} \\
& \left.\lesssim \|\left\{\left[\psi_{l-1}(\mathcal{B})+\psi_{l}(\mathcal{B})+\psi_{l+1}(\mathcal{B})\right] f\right\}_{l=0}^{\infty}\right)\left\|_{L^{1}\left(\ell^{q}\right)} \lesssim\right\| f \|_{F_{1, q}^{0}(G) .}
\end{aligned}
$$

By having observed that Lemmas 4.1 and 4.2 imply Corollary 4.3, and that these lemmas are enough for proving Theorem 3.1, we will proceed with their proofs. For this, let us recall that on any amenable topological group, and also in several spaces of homogeneous type one has the Calderón-Decomposition lemma.

Remark 4.5 For any non-negative function $f \in L^{1}(G)$, one has its Calderón-Zygmund decomposition. Indeed, by following Hebish [31], (whose construction remains valid for any amenable group, in particular, compact Lie groups) one can obtain a suitable family of disjoint open sets $\left\{I_{j}\right\}_{j=0}^{\infty}$ such that

- $f(x) \leq t$, for a.e. $x \in G \backslash \cup_{j \geq 0} I_{j}$,

- $\sum_{j \geq 0}\left|I_{j}\right| \leq \frac{C}{t}\|f\|_{L^{1}(G)}$, and

- $t\left|I_{j}\right| \leq \int_{I_{j}} f(x) d x \leq 2\left|I_{j}\right| t$, for all $j$. 
Now, for every $j \in \mathbb{N}_{0}$, let us define $R_{j}$ by

$$
R_{j}:=\sup \left\{R>0: B\left(z_{j}, R\right) \subset I_{j} \text {, for some } z_{j} \in I_{j}\right\},
$$

where $B\left(z_{j}, R\right)=\left\{x \in I_{j}:\left|z_{j}^{-1} x\right|<R\right\}$. Every $I_{j}$ is bounded, and one can assume that $I_{j} \subset B\left(z_{j}, 2 R_{j}\right)$, where $z_{j} \in I_{j}$. Let us note that by assuming $f\left(e_{G}\right)>t$, (this can be done, just by re-defining $f \in L^{1}(G)$ at the identity element $e_{G}$ of $\left.G\right)$ we should have that

$$
e_{G} \in \bigcup_{j} I_{j}
$$

because $f(x) \leq t$, for a.e. $x \in G \backslash \cup_{j \geq 0} I_{j}$.

Let us define, for every $x \in I_{j}$,

$$
g(x):=\frac{1}{\left|I_{j}\right|} \int_{I_{j}} f(y) d y, \quad b(x)=f(x)-g(x),
$$

and for $x \in G \backslash \cup_{j \geq 0} I_{j}, g(x)=f(x), b(x)=0$. Then, one has the decomposition $f=g+b$, with $b$ having null average on $I_{j}$.

Proof of Lemma 4.1 Let us fix $f \in L^{1}(G)$ to be a non-negative function, and let us consider its Calderón-Zygmund decomposition $f=g+b$ as in Remark 4.5. It is enough to demonstrate that the linear operators $W_{\ell}, \ell \in \mathbb{N}_{0}$, are uniformly bounded on $L^{q}(G), 1<q<\infty$. This fact is straightforward if $q=2$, so it is suffices (by the duality argument) that the operators $W_{\ell}$ are uniformly bounded from $L^{1}(G)$ into $L^{1, \infty}(G)$. So, we will prove the existence of $C>0$, independent of $f \in L^{1}(G)$, and $\ell \in \mathbb{N}_{0}$, such that

$$
\left|\left\{x \in G:\left|W_{\ell} f(x)\right|>t\right\}\right| \leq \frac{C}{t}\|f\|_{L^{1}(G)} .
$$

Let us remark that for every $x \in I_{j}$,

$$
|g(x)|=\left|\frac{1}{\left|I_{j}\right|} \int_{I_{j}} f(y) d y\right| \leq 2 t .
$$

By the Minkowski inequality, we have

$$
\begin{aligned}
& \left|\left\{x \in G:\left|W_{\ell} f(x)\right|>t\right\}\right| \leq\left|\left\{x \in G:\left|W_{\ell} g(x)\right|>\frac{t}{2}\right\}\right| \\
& +\left|\left\{x \in G:\left|W_{\ell} b(x)\right|>\frac{t}{2}\right\}\right| .
\end{aligned}
$$

In view of the Chebyshev inequality, we get 


$$
\begin{aligned}
& \left|\left\{x \in G:\left|W_{\ell} f(x)\right|>t\right\}\right| \\
& \leq\left|\left\{x \in G:\left|W_{\ell} g(x)\right|>\frac{t}{2}\right\}\right|+\left|\left\{x \in G:\left|W_{\ell} b(x)\right|>\frac{t}{2}\right\}\right| \\
& =\left|\left\{x \in G:\left|W_{\ell} g(x)\right|^{2}>\frac{t^{2}}{2^{2}}\right\}\right|+\left|\left\{x \in G:\left|W_{\ell} b(x)\right|>\frac{t}{2}\right\}\right| \\
& \leq \frac{2^{2}}{t^{2}} \int_{G}\left|W_{\ell} g(x)\right|^{2} d x+\left|\left\{x \in G:\left|W_{\ell} b(x)\right|>\frac{t}{2}\right\}\right| \\
& \leq \frac{2^{2}}{t^{2}} \sup _{\ell}\left\|W_{\ell}\right\|_{\mathscr{B}\left(L^{2}(G)\right)} \int_{G}|g(x)|^{2} d x+\left|\left\{x \in G:\left|W_{\ell} b(x)\right|>\frac{t}{2}\right\}\right| \\
& \lesssim \frac{2^{2}}{t^{2}} \int_{G}|g(x)|^{2} d x+\left|\left\{x \in G:\left|W_{\ell} b(x)\right|>\frac{t}{2}\right\}\right|,
\end{aligned}
$$

in view of the $L^{2}(G)$-boundedness of $A$ and the fact that the operators $\psi_{\ell}(\mathcal{B})$ are $L^{2}(G)$ -bounded uniformly in $\ell \in \mathbb{N}_{0}$. Additionally, note that the estimate

$$
\begin{aligned}
\|g\|_{L^{2}(G)}^{2} & =\int_{G}|g(x)|^{2} d x=\sum_{j} \int_{I_{j}}|g(x)|^{2} d x+\int_{G \backslash \cup_{j} I_{j}}|g(x)|^{2} d x \\
& =\sum_{j} \int_{I_{j}}|g(x)|^{2} d x+\int_{G \backslash \cup_{j} I_{j}}|f(x)|^{2} d x \\
& \leq \sum_{j} \int_{I_{j}}(2 t)^{2} d x+\int_{G \backslash \cup_{j} I_{j}} f(x)^{2} d x \lesssim t^{2} \sum_{j}\left|I_{j}\right|+\int_{G \backslash \cup_{j} I_{j}} f(x) f(x) d x \\
& \leq t^{2} \times \frac{C}{t}\|f\|_{L^{1}(G)}+t \int_{G \backslash \cup_{j} I_{j}} f(x) d x \lesssim t\|f\|_{L^{1}(G)},
\end{aligned}
$$

implies that

$$
\left|\left\{x \in G:\left|W_{\ell} f(x)\right|>t\right\}\right| \leq \frac{4}{t}\|f\|_{L^{1}(G)}+\left|\left\{x \in G:\left|W_{\ell} b(x)\right|^{2}>\frac{t}{2}\right\}\right| .
$$

Taking into account that $b \equiv 0$ on $G \backslash \cup_{j} I_{j}$, we have that

$$
b=\sum_{k} b_{k}, b_{k}(x)=b(x) \cdot 1_{I_{k}}(x) .
$$

Let us assume that $I_{j}^{*}$ is an open set, such that $I_{j} \subset I_{j}^{*}$, and $\left|I_{j}^{*}\right|=K\left|I_{j}\right|$ for some $K>0$, and $\operatorname{dist}\left(\partial I_{j}^{*}, \partial I_{j}\right) \geq 4 c \operatorname{dist}\left(\partial I_{j}, e_{G}\right)$, where $c>0$ and $e_{G}$ is the identity element of $G$. So, by the Minkowski inequality we have 


$$
\begin{aligned}
& \left|\left\{x \in G:\left|W_{\ell} b(x)\right|>\frac{t}{2}\right\}\right| \\
& =\left|\left\{x \in \cup_{j} I_{j}^{*}:\left|W_{\ell} b(x)\right|>\frac{t}{2}\right\}\right|+\left|\left\{x \in G \backslash \cup_{j} I_{j}^{*}:\left|W_{\ell} b(x)\right|>\frac{t}{2}\right\}\right| \\
& \leq\left|\left\{x \in G: x \in \cup_{j} I_{j}^{*}\right\}\right|+\left|\left\{x \in G \backslash \cup_{j} I_{j}^{*}:\left|W_{\ell} b(x)\right|>\frac{t}{2}\right\}\right| .
\end{aligned}
$$

Consequently, we deduce the estimates

$$
\begin{aligned}
& \left|\left\{x \in G:\left|W_{\ell} b(x)\right|>\frac{t}{2}\right\}\right| \leq \sum_{j}\left|I_{j}^{*}\right|+\left|\left\{x \in G \backslash \cup_{j} I_{j}^{*}:\left|W_{\ell} b(x)\right|>\frac{t}{2}\right\}\right| \\
& =K \sum_{j}\left|I_{j}\right|+\left|\left\{x \in G \backslash \cup_{j} I_{j}^{*}:\left|W_{\ell} b(x)\right|>\frac{t}{2}\right\}\right| \\
& \leq \frac{C K}{t}\|f\|_{L^{1}(G)}+\left|\left\{x \in G \backslash \cup_{j} I_{j}^{*}:\left|W_{\ell} b(x)\right|>\frac{t}{2}\right\}\right| .
\end{aligned}
$$

Now, using the Chebyshev inequality to estimate the right hand side above we obtain

$$
\begin{aligned}
& \left|\left\{x \in G \backslash \cup_{j} I_{j}^{*}:\left|W_{\ell} b(x)\right|>\frac{t}{2}\right\}\right| \leq \frac{2}{t} \int_{G \backslash \cup_{j} I_{j}^{*}}\left|W_{\ell} b(x)\right| d x \\
& \leq \frac{2}{t} \sum_{k} \int_{G \backslash \cup_{j} I_{j}^{*}}\left|W_{\ell} b_{k}(x)\right| d x .
\end{aligned}
$$

From now, let us denote by $\kappa_{\ell}$ the right convolution kernel of $W_{\ell}:=A \psi_{\ell}(\mathcal{B})$. Observe that

$$
\begin{aligned}
& \left|\left\{x \in G \backslash \cup_{j} I_{j}^{*}:\left|W_{\ell} b(x)\right|>\frac{t}{2}\right\}\right| \leq \frac{2}{t} \sum_{k} \int_{G \backslash \cup_{j} I_{j}^{*}}\left|W_{\ell} b_{k}(x)\right| d x \\
& =\frac{2}{t} \sum_{k} \int_{G \backslash \cup_{j} I_{j}^{*}}\left|b_{k} * \kappa_{\ell}(x)\right| d x \\
& =\frac{2}{t} \sum_{k} \int_{G \backslash \cup_{j} I_{j}^{*}}\left|\int_{I_{k}} b_{k}(z) \kappa_{\ell}\left(z^{-1} x\right) d z\right| d x .
\end{aligned}
$$

By using that the average of $b_{k}$ on $I_{k}$ is zero, $\int_{I_{k}} b_{k}(z) d z=0$, we have 


$$
\begin{aligned}
\frac{2}{t} \sum_{k} \int_{G \backslash \cup_{j} I_{j}^{*}}\left|\int_{I_{k}} b_{k}(z) \kappa_{\ell}\left(z^{-1} x\right) d z\right| d x \\
=\frac{2}{t} \sum_{k} \int_{G \backslash \cup_{j} I_{j}^{*}}\left|\int_{I_{k}} b_{k}(z) \kappa_{\ell}\left(z^{-1} x\right) d z-\kappa_{\ell}(x) \int_{I_{k}} b_{k}(z) d z\right| d x \\
=\frac{2}{t} \sum_{k} \int_{G \backslash \cup_{j} I_{j}^{*}}\left|\int_{I_{k}}\left(\kappa_{\ell}\left(z^{-1} x\right)-\kappa_{\ell}(x)\right) b_{k}(z) d z\right| d x .
\end{aligned}
$$

Assuming the following uniform estimate

$$
M=\sup _{k} \sup _{z \in I_{k}} \sum_{\ell=0}^{\infty} \int_{G \backslash \cup_{j} I_{j}^{*}}\left|\kappa_{\ell}\left(z^{-1} x\right)-\kappa_{\ell}(x)\right| d x<\infty,
$$

we have

$$
\begin{aligned}
& \left|\left\{x \in G \backslash \cup_{j^{\prime}} I_{j}^{*}:\left|W_{\ell} b(x)\right|>\frac{t}{2}\right\}\right| \leq \frac{2 M}{t} \sum_{k} \int_{I_{k}}\left|b_{k}(z)\right| d z \\
& =\frac{2 M}{t}\|b\|_{L^{1}(G)} \leq \frac{6 M}{t}\|f\|_{L^{1}(G)} .
\end{aligned}
$$

So, if we prove the uniform estimate (4.7), we obtain the weak $(1,1)$ inequality for $f \in L^{1}(G), f \geq 0$. For the proof of (4.7) let us use the estimates of the Calderón-Zygmund kernel of every operator $W_{\ell}$. Before continuing with the proof let us use the following geometrical property of the open sets $I_{j}, j \geq 0$ :

Lemma 4.6 Let us consider (4.3). Then, with the notation above, for any $x \in G \backslash \cup_{j} I_{j}^{*}$, and for all $z \in I_{k}$, we have $|z| \lesssim|x|$, i.e. for some $c>0,4 c|z| \leq|x|$.

We will postpone the proof of Lemma 4.6 for a moment to continue with the proof of Lemma 4.1, in order to use the estimate $4 c|z| \leq|x|$, for $x \in G \backslash \cup_{j} I_{j}^{*}, z \in I_{k}$, together with (3.9) in Lemma 3.3 for $\ell \geq 0$, as follows,

$$
\begin{aligned}
M_{k} & :=\sup _{z \in I_{k}} \sum_{\ell=0}^{\infty} \int_{G \backslash \cup_{j} I_{j}^{*}}\left|\kappa_{\ell}\left(z^{-1} x\right)-\kappa_{\ell}(x)\right| d x \lesssim \sup _{z \in I_{k}} \sum_{\ell=0}^{\infty} \int_{|x| \geq 4 c|z|}\left|\kappa_{\ell}\left(z^{-1} x\right)-\kappa_{\ell}(x)\right| d x \\
& \lesssim \sum_{\ell=0}^{\infty} 2^{-\ell\left(s-\frac{n}{2}\right)}\|\sigma\|_{\text {l.u., } L_{s}^{2}(\hat{G})}^{\prime} .
\end{aligned}
$$

The estimate above implies that

$$
M_{k}:=\sup _{z \in I_{k}} \sum_{\ell=0}^{\infty} \int_{G \backslash \cup_{j} I_{j}^{*}}\left|\kappa_{\ell}\left(z^{-1} x\right)-\kappa_{\ell}(x)\right| d x \lesssim\|\sigma\|_{\text {l.u., } L_{s}^{2}(\widehat{G})}^{\prime} .
$$


So, we have (4.5) for $f \in L^{1}(G)$ with $f \geq 0$. Note that if $f \in L^{1}(G)$ is real-valued, one can decompose $f=f^{+}-f^{-}$, as the difference of two non-negative functions, where $f^{+}, f^{-} \in L^{1}(G)$, and $|f|=f^{+}+f^{-}$. Because $f^{+}, f^{-} \leq|f|$, we have

$$
\begin{aligned}
\left|\left\{x \in G:\left|W_{\ell} f(x)\right|>t\right\}\right| & \leq\left|\left\{x \in G:\left|W_{\ell} f_{+}(x)\right|>\frac{t}{2}\right\}\right|+\left|\left\{x \in G:\left|W_{\ell} f_{-}(x)\right|>\frac{t}{2}\right\}\right| \\
& \leq \frac{C}{t}\left\|f_{+}\right\|_{L^{1}(G)}+\frac{C}{t}\left\|f_{-}\right\|_{L^{1}(G)} \\
& \leq \frac{2 C}{t}\|f\|_{L^{1}(G)} .
\end{aligned}
$$

A similar analysis, by splitting a complex function $f \in L^{1}(G)$ into its real and imaginary parts allows to conclude the weak $(1,1)$ inequality $(4.5)$ to complex functions. Thus, the proof of Lemma 4.1 would be complete if we prove Lemma 4.6. That lemma was proved in [8, Page 17] for graded Lie groups and the same proof applies for any amenable group (see Hebish [31]), we will present the proof here for the case of a compact Lie group $G$ for completeness.

Proof of Lemma 4.6 In view of the estimate $\operatorname{dist}\left(\partial I_{j}^{*}, \partial I_{j}\right) \geq 4 c \operatorname{dist}\left(\partial I_{j}, e_{G}\right)$, we will prove that for $x \in G \backslash \cup_{j} I_{j}^{*}$, and all $z \in I_{k}, 4 c|z|=4 c \times \operatorname{dist}\left(z, e_{G}\right) \lesssim \operatorname{dist}\left(\partial I_{k}^{*}, \partial I_{k}\right) \leq|x|$. Indeed, fix $\varepsilon>0$, and let us take $w \in \partial I_{k}$, and $w^{\prime} \in \partial I_{k}^{*}$ such that $d\left(w, w^{\prime}\right) \leq \operatorname{dist}\left(\partial I_{k}, \partial I_{k}^{*}\right)+\varepsilon$. Then, from the triangle inequality, we have

$$
\begin{aligned}
& d\left(z, e_{G}\right) \\
& \leq d(z, w)+d\left(w, w^{\prime}\right)+d\left(w^{\prime}, e_{G}\right) \leq \operatorname{diam}\left(I_{k}\right)+\operatorname{dist}\left(\partial I_{k}, \partial I_{k}^{*}\right)+\operatorname{dist}\left(\partial I_{k}^{*}, e_{G}\right)+\varepsilon \\
& \lesssim \operatorname{diam}\left(I_{k}\right)+\operatorname{dist}\left(\partial I_{k}, \partial I_{k}^{*}\right)+\operatorname{dist}\left(\partial I_{k}, e_{G}\right)+\varepsilon \\
& \lesssim \operatorname{diam}\left(I_{k}\right)+\operatorname{dist}\left(\partial I_{k}, \partial I_{k}^{*}\right)+\frac{1}{4 c} \operatorname{dist}\left(\partial I_{k}, \partial I_{k}^{*}\right)+\varepsilon \\
& \asymp \operatorname{dist}\left(\partial I_{k}, \partial I_{k}^{*}\right)+\varepsilon,
\end{aligned}
$$

where in the last line we have assumed that $\operatorname{diam}\left(I_{k}\right) \asymp \operatorname{dist}\left(\partial I_{k}, \partial I_{k}^{*}\right)$, (with constants of proportionality independent in $k$ ) and that $\operatorname{dist}\left(\partial I_{k}, \partial I_{k}^{*}\right)$ is proportional to $R_{k}$ in view of the relation $\left|I_{k}^{*}\right|=K\left|I_{k}\right|$. Assuming (4.9), one has that for all $\varepsilon>0, d\left(z, e_{G}\right) \lesssim \operatorname{dist}\left(\partial I_{k}, \partial I_{k}^{*}\right)+\varepsilon$, which implies that

$$
d\left(z, e_{G}\right) \lesssim \operatorname{dist}\left(\partial I_{k}, \partial I_{k}^{*}\right) .
$$

To show that the proportionality constant in (4.10) is uniform in $k$, let us recall the definition of the radii $R_{k}^{\prime} s$ in (4.2), that $B\left(z_{k}, R_{k}\right) \subset I_{k} \subset B\left(z_{k}, 2 R_{k}\right)$, and that $B\left(z_{k}, R_{k} / C\right) \subset I_{k}^{*} \subset B\left(z_{k}, C R_{k}\right)$ for some $C>2$ independent of $k$, where for any $k, z_{k} \in I_{k}$. From this remark observe that:

- The condition $B\left(z_{k}, R_{k}\right) \subset I_{k} \subset B\left(z_{k}, 2 R_{k}\right)$, implies that $2 R_{k} \leq \operatorname{diam}\left(I_{k}\right) \leq 4 R_{k}$.

- That $B\left(z_{k}, R_{k}\right) \subset I_{k} \subset I_{k}^{*} \subset B\left(z_{k}, C R_{k}\right)$, implies that

$$
\operatorname{dist}\left(\partial I_{k}, \partial I_{k}^{*}\right) \leq \operatorname{dist}\left(\partial B\left(z_{k}, R_{k}\right), \partial B\left(z_{k}, C R_{k}\right)\right)=(C-1) R_{k} .
$$

On the other hand, by observing that in every step above we can replace $I_{k}^{*}:=B\left(z_{k}, C R_{k}\right)$, in view of the inclusion

$$
I_{k} \subset B\left(z_{k}, 2 R_{k}\right) \subset I_{k}^{*}:=B\left(z_{k}, C R_{k}\right),
$$


we have

$$
(C-2) R_{k}=\operatorname{dist}\left(\partial I_{k}^{*}, \partial B\left(z_{k}, 2 R_{k}\right)\right) \leq \operatorname{dist}\left(\partial I_{k}, \partial I_{k}^{*}\right) .
$$

Consequently,

$$
\begin{aligned}
\operatorname{diam}\left(I_{k}\right) & \asymp R_{k} \asymp \operatorname{dist}\left(\partial B\left(z_{k}, 2 R_{k}\right), \partial B\left(z_{k}, C R_{k}\right)\right) \\
& \asymp \operatorname{dist}\left(\partial I_{k}, \partial I_{k}^{*}\right) .
\end{aligned}
$$

To show that $\operatorname{dist}\left(\partial I_{k}^{*}, \partial I_{k}\right) \leq|x|$, observe that from Remark 4.3, $e_{G} \in \cup_{j} I_{j}$, and because of $x \in G \backslash \cup_{j} I_{j}$,

$$
\operatorname{dist}\left(\partial I_{k}^{*}, \partial I_{k}\right) \lesssim \operatorname{diam}\left(\cup_{j} I_{j}\right) \lesssim d\left(x, e_{G}\right)=|x| .
$$

So, we have guaranteed the existence of a positive constant, which we again denote by $c>0$, such that, $\left\{x \in G: x \in G \backslash \cup_{j} I_{j}^{*}\right\} \subset\left\{x \in G\right.$ : for all $\left.z \in I_{k}, 4 c|z| \leq|x|\right\}$.

Proof of Lemma 4.2 Now, we claim that

$$
W: L^{1}\left(G, \ell^{r}\left(\mathbb{N}_{0}\right)\right) \rightarrow L^{1, \infty}\left(G, \ell^{r}\left(\mathbb{N}_{0}\right)\right), \quad 1<r<\infty .
$$

extends to a bounded operator. For the proof of (4.11), we need to show that there exists a constant $C>0$ independent of $\left\{f_{\ell}\right\} \in L^{1}\left(G, \ell^{r}\left(\mathbb{N}_{0}\right)\right)$ and $t>0$, such that

$$
\left|\left\{x \in G:\left(\sum_{\ell=0}^{\infty}\left|W_{\ell} f_{\ell}(x)\right|^{r}\right)^{\frac{1}{r}}>t\right\}\right| \leq \frac{C}{t}\left\|\left\{f_{\ell}\right\}\right\|_{L^{1}\left(G, \ell^{r}\left(\mathbb{N}_{0}\right)\right.} .
$$

So, fix $\left\{f_{\ell}\right\} \in L^{1}\left(G, \ell^{r}\left(\mathbb{N}_{0}\right)\right)$ and $t>0$, and let $h(x):=\left(\sum_{\ell=0}^{\infty}\left|f_{\ell}(x)\right|^{r}\right)^{\frac{1}{r}}$. Apply the Calderón-Zygmund decomposition Lemma to $h \in L^{1}(G)$ in Remark 4.5 in order to obtain a disjoint collection $\left\{I_{j}\right\}_{j=0}^{\infty}$ of disjoint open sets such that

- $h(x) \leq t$, for a.e. $x \in G \backslash \cup_{j \geq 0} I_{j}$,

- $\quad \sum_{j \geq 0}\left|I_{j}\right| \leq \frac{C}{t}\|h\|_{L^{1}(G)}$, and

- $t \leq \frac{1}{\left|I_{j}\right|} \int_{I_{j}} h(\stackrel{t}{x}) d x \leq 2 t$, for all $j$.

Now, we will define a suitable decomposition of $f_{\ell}$, for every $\ell \geq 0$. Recall that every $I_{j}$ is bounded, and that $I_{j} \subset B\left(z_{j}, 2 R_{j}\right.$ ), where $z_{j} \in I_{j}$ (see Remark 4.5). Let us define, for every $\ell$, and $x \in I_{j}$,

$$
g_{\ell}(x):=\frac{1}{\left|I_{j}\right|} \int_{I_{j}} f_{\ell}(y) d y, \quad b_{\ell}(x)=f_{\ell}(x)-g_{\ell}(x) .
$$

and for $x \in G \backslash \cup_{j \geq 0} I_{j}$,

$$
g_{\ell}(x)=f_{\ell}(x), \quad b_{\ell}(x)=0 .
$$

So, for a.e. $x \in G, \quad f_{\ell}(x)=g_{\ell}(x)+b_{\ell}(x)$. Note that for every $1<r<\infty$, $\left\|\left\{g_{\ell}\right\}\right\|_{L^{r}\left(\ell^{r}\right)}^{r} \leq t^{r-1}\left\|\left\{f_{\ell}\right\}\right\|_{L^{1}\left(\ell^{r}\right)}$. Indeed, for $x \in I_{j}$, Minkowski integral inequality gives, 


$$
\begin{aligned}
\left(\sum_{\ell=0}^{\infty}\left|g_{\ell}(x)\right|^{r}\right)^{\frac{1}{r}} & \leq\left(\sum_{\ell=0}^{\infty}\left|\frac{1}{\left|I_{j}\right|} \int_{I_{j}} f_{\ell}(y) d y\right|^{r} \leq \frac{1}{\left|I_{j}\right|} \int_{I_{j}}\left(\sum_{\ell=0}^{\infty}\left|f_{\ell}(y)\right|^{r}\right)^{\frac{1}{r}} d y\right. \\
& =\frac{1}{\left|I_{j}\right|} \int_{I_{j}} h(y) d y \\
& \leq 2 t .
\end{aligned}
$$

Consequently,

$$
\sum_{\ell=0}^{\infty}\left|g_{\ell}(x)\right|^{r} \leq(2 t)^{r},
$$

and from the fact that $h(x) \leq t$, for a.e. $x \in G \backslash \cup_{j \geq 0} I_{j}$, we have

$$
\begin{aligned}
\left\|\left\{g_{\ell}\right\}\right\|_{L^{r}\left(\ell^{r}\right)}^{r} & =\int_{G} \sum_{\ell=0}^{\infty}\left|g_{\ell}(x)\right|^{r} d x=\sum_{j} \int_{I_{j}} \sum_{\ell=0}^{\infty}\left|g_{\ell}(x)\right|^{r} d x+\int_{G \backslash \cup_{j} I_{j}} \sum_{\ell=0}^{\infty}\left|g_{\ell}(x)\right|^{r} d x \\
& =\sum_{j} \int_{I_{j}} \sum_{\ell=0}^{\infty}\left|g_{\ell}(x)\right|^{r} d x+\int_{G \backslash \cup_{j} I_{j}} \sum_{\ell=0}^{\infty}\left|f_{\ell}(x)\right|^{r} d x \\
& \leq \sum_{j} \int_{I_{j}}(2 t)^{r} d x+\int_{G \backslash \cup_{j} I_{j}} h(x)^{r} d x \\
& \lesssim t^{r} \sum_{j}\left|I_{j}\right|+\int_{G \backslash \cup_{j} I_{j}} h(x)^{r-1} h(x) d x \\
& \leq t^{r} \times \frac{C}{t}\|h\|_{L^{1}(G)}+t^{r-1} \int_{G \backslash \cup_{j} I_{j}} h(x) d x \lesssim t^{r-1}\|h\|_{L^{1}(G)} \\
& =t^{r-1}\left\|\left\{f_{\ell}\right\}\right\|_{L^{1}(\ell)} .
\end{aligned}
$$

Now, by using the Minkowski and the Chebyshev inequality, we obtain

$$
\begin{aligned}
& \left|\left\{x \in G:\left(\sum_{\ell=0}^{\infty}\left|W_{\ell} f_{\ell}(x)\right|^{r}\right)^{\frac{1}{r}}>t\right\}\right| \\
& \leq\left|\left\{x \in G:\left(\sum_{\ell=0}^{\infty}\left|W_{\ell} g_{\ell}(x)\right|^{r}\right)^{\frac{1}{r}}>\frac{t}{2}\right\}\right|+\left|\left\{x \in G:\left(\sum_{\ell=0}^{\infty}\left|W_{\ell} b_{\ell}(x)\right|^{r}\right)^{\frac{1}{r}}>\frac{t}{2}\right\}\right| \\
& \leq \frac{2^{r}}{t^{r}} \int_{G} \sum_{\ell=0}^{\infty}\left|W_{\ell} g_{\ell}(x)\right|^{r} d x+\left|\left\{x \in G:\left(\sum_{\ell=0}^{\infty}\left|W_{\ell} b_{\ell}(x)\right|^{r}\right)^{\frac{1}{r}}>\frac{t}{2}\right\}\right| .
\end{aligned}
$$


In view of Lemma 4.1, $W: L^{r}\left(G, \ell^{r}\left(\mathbb{N}_{0}\right)\right) \rightarrow L^{r}\left(G, \ell^{r}\left(\mathbb{N}_{0}\right)\right)$, extends to a bounded operator and

$$
\int_{G} \sum_{\ell=0}^{\infty}\left|W_{\ell} g_{\ell}(x)\right|^{r} d x=\left\|W\left\{g_{\ell}\right\}\right\|_{L^{r}\left(\ell^{r}\right)}^{r} \lesssim\left\|\left\{g_{\ell}\right\}\right\|_{L^{r}\left(\ell^{r}\right)}^{r} \leq t^{r-1}\left\|\left\{f_{\ell}\right\}\right\|_{L^{1}\left(\ell^{r}\right)}
$$

Consequently,

$$
\begin{aligned}
& \left|\left\{x \in G:\left(\sum_{\ell=0}^{\infty}\left|W_{\ell} f_{\ell}(x)\right|^{r}\right)^{\frac{1}{r}}>t\right\}\right| \\
& \lesssim \frac{1}{t}\left\|\left\{f_{\ell}\right\}\right\|_{L^{1}\left(\ell^{r}\right)}+\mid\left\{x \in G:\left(\sum_{\ell=0}^{\infty}\left|W_{\ell} b_{\ell}(x)\right|^{r}\right)^{\frac{1}{r}}>\frac{t}{2}\right\} .
\end{aligned}
$$

Now, we only need to prove that

$$
\left|\left\{x \in G:\left(\sum_{\ell=0}^{\infty}\left|W_{\ell} b_{\ell}(x)\right|^{r}\right)^{\frac{1}{r}}>\frac{t}{2}\right\}\right| \lesssim \frac{1}{t}\left\|\left\{f_{\ell}\right\}\right\|_{L^{1}\left(\ell^{r}\right)} .
$$

Taking into account that $b_{\ell} \equiv 0$ on $G \backslash \cup_{j} I_{j}$, we have that

$$
b_{\ell}=\sum_{k} b_{\ell, k}, \quad b_{\ell, k}(x)=b_{\ell}(x) \cdot 1_{I_{k}}(x) .
$$

Let us assume that $I_{j}^{*}$ is a open set, such that $\left|I_{j}^{*}\right|=K\left|I_{j}\right|$ for some $K>0$, and $\operatorname{dist}\left(\partial I_{j}^{*}, \partial I_{j}\right) \geq c \operatorname{dist}\left(\partial I_{j}, e_{G}\right)$, where $e_{G}$ is the identity element of $G$. So, by the Minkowski inequality we have,

$$
\begin{aligned}
& \left|\left\{x \in G:\left(\sum_{\ell=0}^{\infty}\left|W_{\ell} b_{\ell}(x)\right|^{r}\right)^{\frac{1}{r}}>\frac{t}{2}\right\}\right| \\
& =\left|\left\{x \in \cup_{j} I_{j}^{*}:\left(\sum_{\ell=0}^{\infty}\left|W_{\ell} b_{\ell}(x)\right|^{r}\right)^{\frac{1}{r}}>\frac{t}{2}\right\}\right|+\left|\left\{x \in G \backslash \cup_{j} I_{j}^{*}:\left(\sum_{\ell=0}^{\infty}\left|W_{\ell} b_{\ell}(x)\right|^{r}\right)^{\frac{1}{r}}>\frac{t}{2}\right\}\right| \\
& \leq\left|\left\{x \in G: x \in \cup_{j} I_{j}^{*}\right\}\right|+\left|\left\{x \in G \backslash \cup_{j} I_{j}^{*}:\left(\sum_{\ell=0}^{\infty}\left|W_{\ell} b_{\ell}(x)\right|^{r}\right)^{\frac{1}{r}}>\frac{t}{2}\right\}\right| .
\end{aligned}
$$

Taking into account that

$$
\left|\left\{x \in G: x \in \cup_{j} I_{j}^{*}\right\}\right| \leq \sum_{j}\left|I_{j}^{*}\right|,
$$

we get 


$$
\begin{aligned}
& \left|\left\{x \in G:\left(\sum_{\ell=0}^{\infty}\left|W_{\ell} b_{\ell}(x)\right|^{2}\right)^{\frac{1}{2}}>\frac{t}{2}\right\}\right| \\
& \leq \sum_{j}\left|I_{j}^{*}\right|+\left|\left\{x \in G \backslash \cup_{j} I_{j}^{*}:\left(\sum_{\ell=0}^{\infty}\left|W_{\ell} b_{\ell}(x)\right|^{2}\right)^{\frac{1}{2}}>\frac{t}{2}\right\}\right| \\
& =K \sum_{j}\left|I_{j}\right|+\left|\left\{x \in G \backslash \cup_{j} I_{j}^{*}:\left(\sum_{\ell=0}^{\infty}\left|W_{\ell} b_{\ell}(x)\right|^{2}\right)^{\frac{1}{2}}>\frac{t}{2}\right\}\right| \\
& \leq \frac{C K}{t}\|f\|_{L^{1}\left(G, \ell^{r}\right)}+\mid\left\{x \in G \backslash \cup_{j} I_{j}^{*}:\left(\sum_{\ell=0}^{\infty}\left|W_{\ell} b_{\ell}(x)\right|^{2}\right)^{\frac{1}{2}}>\frac{t}{2}\right\} .
\end{aligned}
$$

Observe that the Chebyshev inequality implies

$$
\begin{aligned}
& \left|\left\{x \in G \backslash \cup_{j} I_{j}^{*}:\left(\sum_{\ell=0}^{\infty}\left|W_{\ell} b_{\ell}(x)\right|^{r}\right)^{\frac{1}{r}}>\frac{t}{2}\right\}\right| \\
& \leq \frac{2}{t} \int_{G \backslash \cup_{j} I_{j}^{*}}\left(\sum_{\ell=0}^{\infty}\left|W_{\ell} b_{\ell}(x)\right|^{r}\right)^{\frac{1}{r}} d x \\
& =\frac{2}{t} \int_{G \backslash \cup_{j} I_{j}^{*}}\left(\sum_{\ell=0}^{\infty}\left|\left(W_{\ell}\left(\sum_{k} b_{\ell, k}\right)\right)^{(x)}\right|^{\frac{1}{r}}\right)^{\frac{1}{r}} d x \\
& =\frac{2}{t} \int_{G \backslash \cup_{j} I_{j}^{*}} \|\left\{\left(W_{\ell}\left(\sum_{k} b_{\ell, k}\right)(x)\right\}_{\ell=0}^{\infty} \|_{\ell r\left(\mathbb{N}_{0}\right)} d x\right. \\
& =\frac{2}{t} \int_{G \backslash \cup_{j} I_{j}^{*}}\left\|\left\{\sum_{k}\left(W_{\ell} b_{\ell, k}\right)(x)\right\}_{\ell=0}^{\infty}\right\|_{\ell r\left(\mathbb{N}_{0}\right)} d x \\
& \leq \frac{2}{\sum_{k}} \int_{G \backslash \cup_{j} I_{j}^{*}}\left(\sum_{\ell=0}^{\infty}\left|\left(W_{\ell} b_{\ell, k}\right)(x)\right|^{r}\right)^{\frac{1}{r}} d x .
\end{aligned}
$$

Now, if $\kappa_{\ell}$ is the right convolution Calderón-Zygmund kernel of $W_{\ell}$, and by using that $\int_{I_{k}} b_{k, \ell}(y) d y=0$, we have that 


$$
\begin{aligned}
\left(\sum_{\ell=0}^{\infty}\left|\left(W_{\ell} b_{\ell, k}\right)(x)\right|^{r}\right)^{\frac{1}{r}} & =\left(\sum_{\ell=0}^{\infty}\left|b_{\ell, k} * \kappa_{\ell}(x)\right|^{r}\right)^{\frac{1}{r}} \\
& =\left(\left.\left.\sum_{\ell=0}^{\infty}\left|\int_{I_{k}} \kappa_{\ell}\left(y^{-1} x\right) b_{\ell, k}(y) d y-\kappa_{\ell}(x) \int_{I_{k}} b_{\ell, k}(y) d y\right|^{\frac{1}{r}}\right|^{\frac{1}{r}}\right|^{\frac{1}{r}}\right. \\
& =\left(\sum_{\ell=0}^{\infty}\left|\int_{I_{k}}\left(\kappa_{\ell}\left(y^{-1} x\right)-\kappa_{\ell}(x)\right) b_{\ell, k}(y) d y\right|^{.}\right.
\end{aligned}
$$

Now, we will proceed as follows. By using that $\left|b_{\ell, k}(y)\right|^{r} \leq \sum_{\ell^{\prime}=0}^{\infty}\left|b_{\ell^{\prime}, k}(y)\right|^{r}$, by an application of the Minkowski integral inequality, we have

$$
\begin{aligned}
& \left(\sum_{\ell=0}^{\infty}\left|\left(W_{\ell} b_{\ell, k}\right)(x)\right|^{r}\right)^{\frac{1}{r}}=\left(\sum_{\ell=0}^{\infty}\left|\int_{I_{k}}\left(\kappa_{\ell}\left(y^{-1} x\right)-\kappa_{\ell}(x)\right) b_{\ell, k}(y) d y\right|^{r}\right)^{\frac{1}{r}} \\
& \leq \int_{I_{k}}\left(\sum_{\ell=0}^{\infty}\left|\kappa_{\ell}\left(y^{-1} x\right)-\kappa_{\ell}(x)\right|^{r}\left|b_{\ell, k}(y)\right|^{r}\right)^{\frac{1}{r}} d y \\
& \leq \int_{I_{k}}\left(\sum_{\ell^{\prime}=0}^{\infty}\left|b_{\ell^{\prime}, k}(y)\right|^{r}\right)^{\frac{1}{r}}\left(\sum_{\ell=0}^{\infty}\left|\kappa_{\ell}\left(x y^{-1}\right)-\kappa_{\ell}(x)\right|^{r}\right)^{\frac{1}{r}} d y .
\end{aligned}
$$

Consequently, we deduce,

$$
\begin{aligned}
& \frac{2}{t} \sum_{k} \int_{G \backslash \cup_{j} I_{j}^{*}}\left(\sum_{\ell=0}^{\infty}\left|\left(W_{\ell} b_{\ell, k}\right)(x)\right|^{r}\right)^{\frac{1}{r}} d x \\
& \leq \frac{2}{t} \sum_{k} \int_{G \backslash \cup_{j} I_{j}^{*}} \int\left(\sum_{I_{k}}^{\infty}\left|b_{\ell^{\prime}, k}(y)\right|^{r}\right)^{\frac{1}{r}}\left(\sum_{\ell=0}^{\infty}\left|\kappa_{\ell}\left(y^{-1} x\right)-\kappa_{\ell}(x)\right|^{r}\right)^{\frac{1}{r}} d y d x \\
& =\frac{2}{t} \sum_{k} \int_{I_{k}} \int_{G \backslash \cup_{j} I_{j}^{*}}\left(\sum_{\ell^{\prime}=0}^{\infty}\left|b_{\ell^{\prime}, k}(y)\right|^{r}\right)^{\frac{1}{r}}\left(\sum_{\ell=0}^{\infty}\left|\kappa_{\ell}\left(y^{-1} x\right)-\kappa_{\ell}(x)\right|^{r}\right)^{\frac{1}{r}} d x d y \\
& =\frac{2}{t} \sum_{k} \int_{I_{k}}\left(\sum_{\ell^{\prime}=0}^{\infty}\left|b_{\ell^{\prime}, k}(y)\right|^{r}\right)_{G \backslash \cup_{j} I_{j}^{*}}^{\frac{1}{r}}\left(\sum_{\ell=0}^{\infty}\left|\kappa_{\ell}\left(y^{-1} x\right)-\kappa_{\ell}(x)\right|^{r}\right)^{\frac{1}{r}} d x d y .
\end{aligned}
$$

Let us recall that for $x \in G \backslash \cup_{j} I_{j}^{*}$, and $y \in I_{k}$, in view of Lemma 4.6, we have that $4 c|y| \leq|x|$. So,

$$
\left\{x \in G: x \in G \backslash \cup_{j} I_{j}^{*}\right\} \subset\left\{x \in G: \text { for all } z \in I_{k}, 4 c|z| \leq|x|\right\} .
$$


Now, in view of (3.9) in Lemma 3.3 with $\ell \geq 0$, we deduce (4.8) and as a consequence we get

$$
\begin{aligned}
& \int_{G \backslash \cup_{j} I_{j}^{*}}\left(\sum_{\ell=0}^{\infty}\left|\kappa_{\ell}\left(y^{-1} x\right)-\kappa_{\ell}(x)\right|^{r}\right)^{\frac{1}{r}} d x \leq \int_{G \backslash \cup_{j} I_{j}^{*}} \sum_{\ell=0}^{\infty}\left|\kappa_{\ell}\left(y^{-1} x\right)-\kappa_{\ell}(x)\right| d x \\
\leq & \sum_{\ell=0}^{\infty} \int_{G \backslash \cup_{j} I_{j}^{*}}\left|\kappa_{\ell}\left(y^{-1} x\right)-\kappa_{\ell}(x)\right| d x \\
\leq & \sum_{\ell=0}^{\infty} \int_{|x|>4 c|y|}\left|\kappa_{\ell}\left(y^{-1} x\right)-\kappa_{\ell}(x)\right| d x \lesssim\|\sigma\|_{l . u ., L_{s}^{2}(\widehat{G})}^{\prime} .
\end{aligned}
$$

Thus, we have proved the estimate

$$
\begin{aligned}
& \left|\left\{x \in G:\left(\sum_{\ell=0}^{\infty}\left|W_{\ell} b_{\ell}(x)\right|^{r}\right)^{\frac{1}{r}}>\frac{t}{2}\right\}\right| \lesssim \frac{2}{t} \sum_{k} \int_{I_{k}}\left(\sum_{\ell^{\prime}=0}^{\infty}\left|b_{\ell^{\prime}, k}(y)\right|^{r}\right)^{\frac{1}{r}} d y \\
& =\frac{2}{t} \int_{\cup_{k} I_{k}}\left(\sum_{\ell^{\prime}=0}^{\infty}\left|b_{\ell^{\prime}}(y)\right|^{r}\right)^{\frac{1}{r}} d y \\
& \lesssim \frac{1}{t}\left\|\left\{f_{\ell}\right\}\right\|_{L^{1}\left(\ell^{r}\right)} .
\end{aligned}
$$

Thus, the proof of the weak $(1,1)$ inequality is complete and we have that

$$
W: L^{1}\left(G, \ell^{r}\left(\mathbb{N}_{0}\right)\right) \rightarrow L^{1, \infty}\left(G, \ell^{r}\left(\mathbb{N}_{0}\right)\right), \quad 1<r<\infty,
$$

admits a bounded extension. The proof of Lemma 4.2 is complete.

Now, having proved Lemma 4.1 and Lemma 4.2, the duality argument in Remark 4.4 proves the Hörmander-Mihlin Theorem 3.1.

Remark 4.7 Let us return to the historical Marcinkiewicz condition on the torus $\mathbb{}$,

$$
\sup _{\xi \in \mathbb{Z}}|\sigma(\xi)|+\sup _{j \in \mathbb{N}_{0}} \sum_{2^{j-1} \leq|\xi|<2^{j}}|\sigma(\xi+1)-\sigma(\xi)|<\infty .
$$

Let $s_{0} \in \mathbb{N}$. For a general compact Lie group $G$ of dimension $n$, one says that a Fourier multiplier $A \equiv T_{\sigma}$ satisfies the (weak) Marcinkiewicz condition of order $s_{0}$, if its symbol $\sigma$ satisfies:

$$
\|\sigma\|_{L^{\infty}(\widehat{G})}+\left\|\sigma \cdot 1_{\left\{[\xi] \in \widehat{G}: 2^{j-1} \leq\langle\xi\rangle<2^{j}\right\}}\right\|_{\dot{L}_{s_{0}}^{1}(\widehat{G})} \lesssim_{s_{0}} 2^{j\left(n-s_{0}\right)}, j \geq 0
$$

uniformly in $j$, with the $\dot{L}_{s_{0}}^{1}(\widehat{G})$-norm defined via: 


$$
\|\tau\|_{\dot{L}_{s_{0}}^{1}(\widehat{G})}:=\sum_{|\alpha|=s_{0}} \sum_{[\xi] \in \widehat{G}} d_{\xi} \operatorname{Tr}\left|\Delta_{\xi}^{\alpha} \tau(\xi)\right|,\left|\Delta_{\xi}^{\alpha} \tau\right|:=\sqrt{\operatorname{Tr}\left(\Delta_{\xi}^{\alpha} \tau\left(\Delta_{\xi}^{\alpha} \tau\right)^{*}\right)}
$$

It was proved in [23], that for $0 \leq s_{0} \leq n$, a symbol satisfying (4.20) also satisfies the Hörmander-Mihlin condition (3.3) for any $s>n / 2$. In view of Theorem 3.1, a Fourier multiplier $A \equiv T_{\sigma}$ satisfying (4.20) extends to a bounded operator from $F_{p, q}^{r}(G)$ into $F_{p, q}^{r}(G)$ for all $1<p, q<\infty$, and all $r \in \mathbb{R}$, and for $p=1, A$ admits a bounded extension from $F_{1, q}^{r}(G)$ into weak- $F_{1, q}^{r}(G)$. In the case of the torus $G=\mathbb{T}, 1<p, q<\infty$, and $n=1=s_{0}$, (4.20)

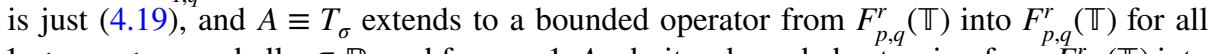
$1<p, q<\infty$, and all $r \in \mathbb{R}$, and for $p=1, A$ admits a bounded extension from $F_{1, q}^{r}(\mathbb{T})$ into weak- $F_{1, q}^{r}(\mathbb{T})$. In view of the Littlewood-Paley theorem, for $1<p<\infty, q=2$ and $r=0$, the previous estimate recovers the classical Marcinkiewicz estimate [35]. We summarise this discussion in the following corollary.

Corollary 4.8 Let us assume that $G$ is a compact Lie group of dimension $n$. Let $\sigma \in \Sigma(\widehat{G})$ be a symbol satisfying

$$
\|\sigma\|_{L^{\infty}(\widehat{G})}+\left\|\sigma \cdot 1_{\left\{[\xi] \in \widehat{G}: 2^{j-1} \leq\langle\xi\rangle<2^{j}\right\}}\right\|_{\dot{L}_{s_{0}}^{1}(\widehat{G})} \lesssim 2^{j\left(n-s_{0}\right)}, j \geq 0,
$$

uniformly in $j$, for some $0 \leq s_{0} \leq n, s_{0} \in \mathbb{N}$. Then $A \equiv T_{\sigma}$ extends to a bounded operator from $F_{p, q}^{r}(G)$ into $F_{p, q}^{r}(G)$ for all $1<p, q<\infty$, and all $r \in \mathbb{R}$. For $p=1, A$ admits $a$ bounded extension from $F_{1, q}^{r}(G)$ into weak- $F_{1, q}^{r}(G)$.

Proof That a symbol satisfying (4.21) for some integer $s_{0}$ with $0 \leq s_{0} \leq n$, satisfies also the Hörmander condition (3.3) for all $s>\frac{n}{2}$ was proved in Theorem 6.16 of [23]. In view of Theorem 3.1 we conclude the proof.

Acknowledgements We would like to thank the anonymous referee for his/her helpful comments.

Open Access This article is licensed under a Creative Commons Attribution 4.0 International License, which permits use, sharing, adaptation, distribution and reproduction in any medium or format, as long as you give appropriate credit to the original author(s) and the source, provide a link to the Creative Commons licence, and indicate if changes were made. The images or other third party material in this article are included in the article's Creative Commons licence, unless indicated otherwise in a credit line to the material. If material is not included in the article's Creative Commons licence and your intended use is not permitted by statutory regulation or exceeds the permitted use, you will need to obtain permission directly from the copyright holder. To view a copy of this licence, visit http://creativecommons.org/licenses/by/4.0/.

\section{References}

1. Alexopoulos, G.: Spectral multipliers on Lie groups of polynomial growth. Proc. Amer. Math. Soc. 120, 973-979 (1994)

2. Anker, J.P.: $L^{p}$-Fourier multipliers on Riemannian symmetric spaces of the noncompact type. Ann. Math. 132, 597-628 (1990)

3. Baernstein, A., II., Sawyer, E.T.: Embedding and multiplier theorems for $H^{p}\left(R^{n}\right)$. Mem. Amer. Math. Soc. 318 (1985)

4. Calderón, A.P., Torchinsky, A.: Parabolic maximal functions associated with a distribution. II. Adv. Math. 24, 101-171 (1977) 
5. Cardona,D.: Besov continuity of pseudo-differential operators on compact Lie groups revisited. C. R. Math. Acad. Sci. Paris Vol. 355, Issue 5, May (2017), Pag. 533-537

6. Cardona, D.: Continuity of pseudo-differential operators on Besov spaces on compact homogeneous manifolds. J. Pseudo-Differ. Oper. Appl. 9(4), 861-880 (2018)

7. Cardona, D., Delgado, J., Ruzhansky, M.: $L^{p}$-bounds for pseudo-differential operators on graded Lie groups. J. Geom. Anal. (2019). https://doi.org/10.1007/s12220-021-00694-1

8. Cardona,D., Ruzhansky, M.:Fourier multipliers for Triebel-Lizorkin spaces on graded Lie groups. arXiv:2101.05856

9. Cardona,D., Ruzhansky,M.: Boundedness of pseudo-differential operators in subelliptic Sobolev and Besov spaces on compact Lie groups. arXiv:1901.06825

10. Cardona,D., Ruzhansky,M.: Subelliptic pseudo-differential operators and Fourier integral operators on compact Lie groups. arXiv:2008.09651

11. Cardona,D., Ruzhansky,M.: Littlewood-Paley theorem, Nikolskii inequality, Besov spaces, Fourier and spectral multipliers on graded Lie groups. arXiv:1610.04701

12. Cardona, D., Ruzhansky, M.: Multipliers for Besov spaces on graded Lie groups. C. R. Math. Acad. Sci. Paris. 4(355), 400-405 (2017)

13. P. Chen, E. M. Ouhabaz, A. Sikora, L. Yan, L, Restriction estimates, sharp spectral multipliers and endpoint estimates for Bochner-Riesz means. arXiv:1202.4052

14. Cowling, M., Sikora, A.: A spectral multiplier theorem for a sublaplacian on SU(2). Math. Z. 238, 1-36 (2001)

15. Delgado, J., Ruzhansky, M.: $L^{p}$-bounds for pseudo-differential operators on compact Lie groups. J. Inst. Math. Jussieu 18(3), 531-559 (2019)

16. Coifman, R.R., De Guzmán, M.: Singular integrals and multipliers on homogeneous spaces. Rev. un. Mat. Argentina, pp. 137-143 (1970)

17. Coifman,R., Weiss, G.:Analyse harmonique non-commutative sur certains espaces homogénes. (French) Étude de certaines intégrales singuliéres. Lecture Notes in Mathematics, Vol. 242. SpringerVerlag, Berlin-New York, 1971. v+160 pp

18. Coifman, R., Weiss, G.: Multiplier transformations of functions on SU(2) and $\Sigma_{2}$. Rev. Un. Mat. Argent. 25, 145-166 (1970)

19. Coifman, R., Weiss, G.: Central multiplier theorems for compact Lie groups. Bull. Am. Math. Soc. 80, 124-126 (1973)

20. ter Elst, A.F.M., Robinson, D.W.: Spectral estimates for positive Rockland operators, in Algebraic groups and Lie groups, Austral. Math. Soc. Lect. Ser., vol. 9, pp. 195-213. Cambridge University Press (1997)

21. Fejér, L.:Sur les fonctions intégrables et bornés, C.R. Acad. Sci. Paris, 10 décembre (1900), 984-987

22. Fefferman, C.: The multiplier problem for the ball. Ann. Math. 2(94), 330-336 (1971)

23. Fischer,V.: Differential structure on the dual of a compact Lie group, arXiv:1610.06348

24. Fischer, V., Ruzhansky, M.: Sobolev spaces on graded groups. Ann. Inst. Fourier 67, 1671-1723 (2017)

25. Fischer,V., Ruzhansky,M.: Fourier multipliers on graded Lie groups. arXiv:1411.6950. Colloq. Math. https://doi.org/10.4064/cm7817-6-2020.

26. Fischer,V., Ruzhansky,M.: Quantization on nilpotent Lie groups. Progress in Mathematics, Vol. 314, Birkhäuser, (2016). (open access book)

27. Fischer, V., Ruzhansky, M.: Sobolev spaces on graded groups. Ann. Inst. Fourier (Grenoble) 67(4), 1671-1723 (2017)

28. Folland, G., Stein, E.: Hardy spaces on homogeneous groups, Mathematical Notes, vol. 28. N.J.; University of Tokyo Press, Tokyo, Princeton University Press, Princeton (1982)

29. Furioli, G., Melzi, C., Veneruso, A.: Littlewood-Paley decompositions and Besov spaces on Lie groups of polynomial growth. Math. Nachr. 279(9-10), 1028-1040 (2006)

30. Grafakos, L.: Some remarks on the Miklhin-Hörmander and Marcinkiewicz multiplier theorems: a short historical account and a recent improvement. J. Geom. Anal., to appear in a special volume in honor of E. M. Stein

31. Hebish,W. : Calderón Zygmund decompositions on amenable groups. arXiv:1810.03566

32. Helffer, B., Nourrigat, J.: Caracterisation des opérateurs hypoelliptiques homogènes invariants à gauche sur un groupe de Lie nilpotent gradué. Comm. Par. Diff. Equ. 4, 899-958 (1979)

33. Hong,Q., Hu,G., Ruzhansky,M.: Fourier multipliers for Hardy spaces on graded Lie groups, preprint

34. Hörmander, L.: Estimates for translation invariant operators in $L^{p}$ spaces. Acta Math. 104, 93-140 (1960)

35. Marcinkiewicz, J.: Sur les multiplicateurs des séries de Fourier. Studia Math. 8, 78-91 (1939) 
36. Mihlin,S. G.: On the multipliers of Fourier integrals. Dokl. Akad. Naulc SSSR (N. S.), 109 (1956), 701-703 (Russian)

37. Nursultanov, E., Ruzhansky, M., Tikhonov, S.: Nikolskii inequality and functional classes on compact Lie groups. Funct. Anal. Appl. 49, 226-229 (2015)

38. Nursultanov,E., Ruzhansky,M., Tikhonov,S.: Nikolskii inequality and Besov, Triebel-Lizorkin, Wiener and Beurling spaces on compact homogeneous manifolds. Ann. Sc. Norm. Super. Pisa Cl. Sci., Vol. XVI, (2016), 981-1017

39. Park, B.: Fourier multiplier theorems for Triebel-Lizorkin spaces. Math. Z. 293, 221-258 (2019)

40. Ruzhansky,M., Turunen,V.: Pseudo-differential Operators and Symmetries: Background Analysis and Advanced Topics Birkhäuser-Verlag, Basel, (2010)

41. Ruzhansky, M., Turunen, V.: Global quantization of pseudo-differential operators on compact Lie groups, SU(2) and 3-sphere. Int. Math. Res. Not. IMRN. 11, 2439-2496 (2013)

42. Ruzhansky, M., Turunen, V., Wirth, J.: Hörmander class of pseudo-differential operators on compact Lie groups and global hypoellipticity. J. Fourier Anal. Appl. 20, 476-499 (2014)

43. Ruzhansky, M., Wirth, J.: Global functional calculus for operators on compact Lie groups. J. Funct. Anal. 267, 144-172 (2014)

44. Ruzhansky, M., Wirth, J.: $L^{p}$ Fourier multipliers on compact Lie groups. Math. Z. 280, 621-642 (2015)

45. Seeger, A.: A limit case of the Hörmander multiplier theorem. Monatsh. Math. 105, 151-160 (1988)

46. Seeger, A.: Estimates near $L^{1}$ for Fourier multipliers and maximal functions. Arch. Math. (Basel) 53, 188-193 (1989)

47. Seeger, A.: Remarks on singular convolution operators. Studia Math. 97, 91-114 (1990)

48. Stein,E. M.: Topics in Harmonic Analysis Related to the Littlewood-Paley Theory, vol. 63 of Annals of Mathematics Studies. Princeton University Press, Princeton (1970)

49. Taibleson, M., Weiss, G.: The molecular characterization of certain Hardy spaces. Astérisque 77, 67-151 (1980)

50. Triebel, H.: Theory of function spaces. Monographs in Mathematics, vol. 78. Birkhäuser Verlag, Basel (1983)

51. Triebel, H.:Theory of function spaces. III, volume 100 of Monographs in Mathematics. Birkhäuser Verlag, Basel, (2006)

52. Varopoulos,N. Th., Saloff-Coste,L., Coulhon,T.: Analysis and geometry on groups. Cambridge Tracts in Mathematics, 100. Cambridge University Press, Cambridge, (1992)

53. Weiss, N.: $L^{p}$ estimates for bi-invariant operators on compact Lie groups. Am. J. Math. 94, 103-118 (1972)

Publisher's Note Springer Nature remains neutral with regard to jurisdictional claims in published maps and institutional affiliations. 